\title{
Transcript analyses reveal a comprehensive role of abscisic acid in modulating fruit ripening in Chinese jujube
}

\author{
Zhong Zhang ${ }^{1,2}$, Chenxuan Kang ${ }^{1}$, Shuyi Zhang ${ }^{1,4}$ and Xingang $\mathrm{Li}^{1,2,3^{*}}$
}

\begin{abstract}
Background: Chinese jujube (Ziziphus jujuba Mill.) is a non-climacteric fruit; however, the underlying mechanism of ripening and the role of abscisic acid involved in this process are not yet understood for this species.

Results: In the present study, a positive correlation between dynamic changes in endogenous ABA and the onset of jujube ripening was determined. Transcript analyses suggested that the expression balance among genes encoding nine-cis-epoxycarotenoid dioxygenase (ZjNCED3), ABA-8'-hydroxylase (ZjCYP707A2), and beta-glucosidase (ZjBG4, ZjBG5, ZjBG8, and $Z j B G 9$ ) has an important role in maintaining $A B A$ accumulation, while the expression of a receptor (ZjPYL8), protein phosphatase 2C (ZjPP2C4-8), and sucrose nonfermenting 1-related protein kinase 2 (ZjSnRK2-2 and ZjSnRK2-5) is important in regulating fruit sensitivity to ABA applications. In addition, white mature 'Dongzao' fruit were harvested and treated with $50 \mathrm{mg} \mathrm{L}^{-1} \mathrm{ABA}$ or $50 \mathrm{mg} \mathrm{L}^{-1}$ nordihydroguaiaretic acid (NDGA) to explore the role of ABA in jujube fruit ripening. By comparative transcriptome analyses, 1103 and 505 genes were differentially expressed in response to ABA and NDGA applications on the 1st day after treatment, respectively. These DEGs were associated with photosynthesis, secondary, lipid, cell wall, and starch and sugar metabolic processes, suggesting the involvement of ABA in modulating jujube fruit ripening. Moreover, ABA also exhibited crosstalk with other phytohormones and transcription factors, indicating a regulatory network for jujube fruit ripening.
\end{abstract}

Conclusions: Our study further elucidated ABA-associated metabolic and regulatory processes. These findings are helpful for improving strategies for jujube fruit storage and for gaining insights into understand complex non-climacteric fruit ripening processes.

Keywords: Chinese jujube (Ziziphus jujuba), Abscisic acid, Fruit ripening, Non-climacteric, Transcriptome sequencing

\section{Background}

Chinese jujube (Ziziphus jujuba Mill.) is a popular fruit crop species that is native to China and is highly desired by consumers worldwide due to the abundant nutritional and health benefits of the fruit $[1,2]$. However, the flesh jujube fruit has a very short shelf-life underlined by rapid dehydration or water-soaking deterioration within 2-3 days after harvest [3]. Therefore, fruit storage and quality maintenance have been among the most urgent challenges in the development of the jujube industry,

\footnotetext{
*Correspondence: xingangle@nwsuaf.edu.cn

${ }^{1}$ College of Forestry, Northwest A\&F University, Yangling 712100, Shaanxi, China

${ }^{2}$ Key Comprehensive Laboratory of Forestry of Shaanxi Province, Northwest A\&F University, Yangling 712100, Shaanxi, China

Full list of author information is available at the end of the article
}

whereas knowledge related to its ripening characterization and regulation is lacking. Over the past few decades, great strides have been made in elucidating the regulation of fruit ripening [4]; in particular, ethylene and abscisic acid (ABA) are recognized as the most important phytohormones that are directly or indirectly involved in the ripening of both climacteric and non-climacteric fruit $[5,6]$. Recently, Chinese jujube has been characterized as a non-climacteric fruit, while a basal level of ethylene is still needed to maintain full fruit maturity [7]. These findings also reveal that the regulation of ripening is relatively complex and that there is a further need to explore these mechanisms to deepen our understanding of the ripening of Chinese jujube fruit.

With regard to $\mathrm{ABA}$, the presence of dramatically increased levels in fruit during the onset of ripening has

(c) The Author(s). 2019 Open Access This article is distributed under the terms of the Creative Commons Attribution 4.0 International License (http://creativecommons.org/licenses/by/4.0/), which permits unrestricted use, distribution, and 
been reported in several fruit crop species, including grape [8], sweet cherry [9], cucumber [10], watermelon [11], and persimmon [5], which points to a role for ABA in triggering the onset of fruit ripening [8]. Moreover, applications of exogenous $\mathrm{ABA}$ and nordihydroguaiaretic acid (NDGA, an inhibitor of ABA biosynthesis) have enabled us to identify ABA-dependent pathways $[12,13]$; increased numbers of research findings have suggested a positive role for $\mathrm{ABA}$ in promoting the metabolism and accumulation of soluble sugars [12, 14], formation of peel color $[15,16]$, and modification of cell wall catabolism [17], thereby accelerating ripening processes [5]. Fruit ripening is a highly integrated process that involves hormone control and crosstalk, as well as alterations to the numbers of transcripts of transcription factors (TFs) $[18,19]$. Increasing amounts of transcriptome sequencing data have provided us with more extensive insight into molecular mechanisms and regulatory networks. For example, ABA regulates the expression of the vast majority of genes involved in strawberry fruit ripening [19], and ABA also has a potential influence on the metabolism and signaling of ethylene, auxin, and gibberellins (GAs) [12, 20-22]. Nevertheless, the involvement of ABA in regulating fruit ripening has scarcely been reported in the non-climacteric fruit of Chinese jujube.

In the present study, we aimed to explore the putative role of $\mathrm{ABA}$ in the regulation of jujube fruit ripening. The dynamic changes in ABA levels during fruit ripening processes were determined, and fruit undergoing the onset of ripening were harvested and treated with exogenous $A B A$ and NDGA to investigate the ABA-dependent pathways and metabolic processes. Using the jujube reference genome (LPXJ00000000.1), we identified genes involved in ABA biosynthesis, metabolism, and signaling (Fig. 1), and their expression during fruit development and ripening was determined by qRT-PCR. In addition, we simultaneously examined ABA-associated ripening metabolism and regulatory networks comprising hormone crosstalk and transcription factor activity via transcriptome sequencing. These results provide insights into the ABA network in non-climacteric ripening and into further improvement strategies for jujube fruit storage.

\section{Results}

Accumulation of endogenous ABA along with Chinese jujube fruit development and ripening

The whole dynamic growth of 'Dongzao' fruit conformed to a double-sigmoidal pattern (Fig. 2), with two rapid growth phases from E2 to E3, followed by E4 to WM. The WM stage was defined as the onset of fruit ripening, during which the fruit color turned whitish-green and the weight accumulation rate clearly decreased. The content of endogenous ABA was determined along with fruit growth processes, with low and stable levels in immature fruit; the content displayed a maximum increase at WM and remained at a high level in BR and HR fruit. Afterwards, the content decreased at the FR stage with a relatively lower level than that observed in the HR fruit. We performed a statistical analysis by using a Person correlation model, and the results revealed a significant correlation between the ABA content and fruit weight, with an $\mathrm{R}$ squared value $=0.9254(p<0.0001$, two tailed $)$.

\section{Relative expression of $A B A$ biosynthesis genes during Chinese jujube fruit development and ripening}

In the ABA biosynthesis pathway, 13 genes were identified from the reference jujube genome, including 1 zeaxanthin epoxidase (ZEP), 3 NCED, 1 xanthoxin dehydrogenase (ABA2), 6 abscisic aldehyde oxidase (AAO), and 2 molybdenum cofactor sulfurtransferase (ABA3) genes. The relative expression of ZjZEP did not significantly change during jujube fruit development and ripening (Fig. 3). ZjNCED1 was consistently expressed during the whole growth process, except at the E1 stage, during which relatively high expression was detected. ZjNCED2 also showed a stable expression pattern, but the level was slightly lower at the HR and FR stages than at the other stages. ZjNCED3 showed rapid increase in expression at the WM stage and maintained a relatively high level during fruit ripening. ZjABA2 was not highly expressed in immature fruit, but the number of transcripts increased significantly after the WM stage. ZjAAO3 was expressed at a low level, but its expression rapidly increased at WM and remained high during ripening, while the relative expression of ZjAAO1, ZjAAO2, ZjAAO4, ZjAAO5, and ZjAAO6 did not correlate with ABA accumulation. In addition, $Z j A B A 3-1$ and $Z j A B A 3-2$ showed a similar trend in which their expression level was higher after the WM stage than in the immature fruit.

\section{Relative expression of ABA metabolism genes during Chinese jujube fruit development and ripening}

In the ABA catabolism pathway, we identified five CYP707As. The relative expression of ZjCYP707A1 was low and did not significantly change during fruit development and ripening (Fig. 4). ZjCYP707A2 exhibited relatively low expression in immature fruit; however, its transcription strongly increased, which was accompanied by ABA accumulation, and reached a maximum level at the FR stage. ZjCYP707A3 showed relatively high expression in E1 fruit, but this expression decreased to a very low level afterwards. ZjCYP707A4 was highly expressed only at the YF stage. ZjCYP707A5 had a relatively high expression at the YF stage, but the expression decreased afterward, with no clear difference.

In the ABA homeostasis pathway, $8 B G$ s were identified. Among them, ZjBG4, ZjBG5, ZjBG8, and ZjBG9 had similar expression patterns along with $A B A$ accumulation; the 


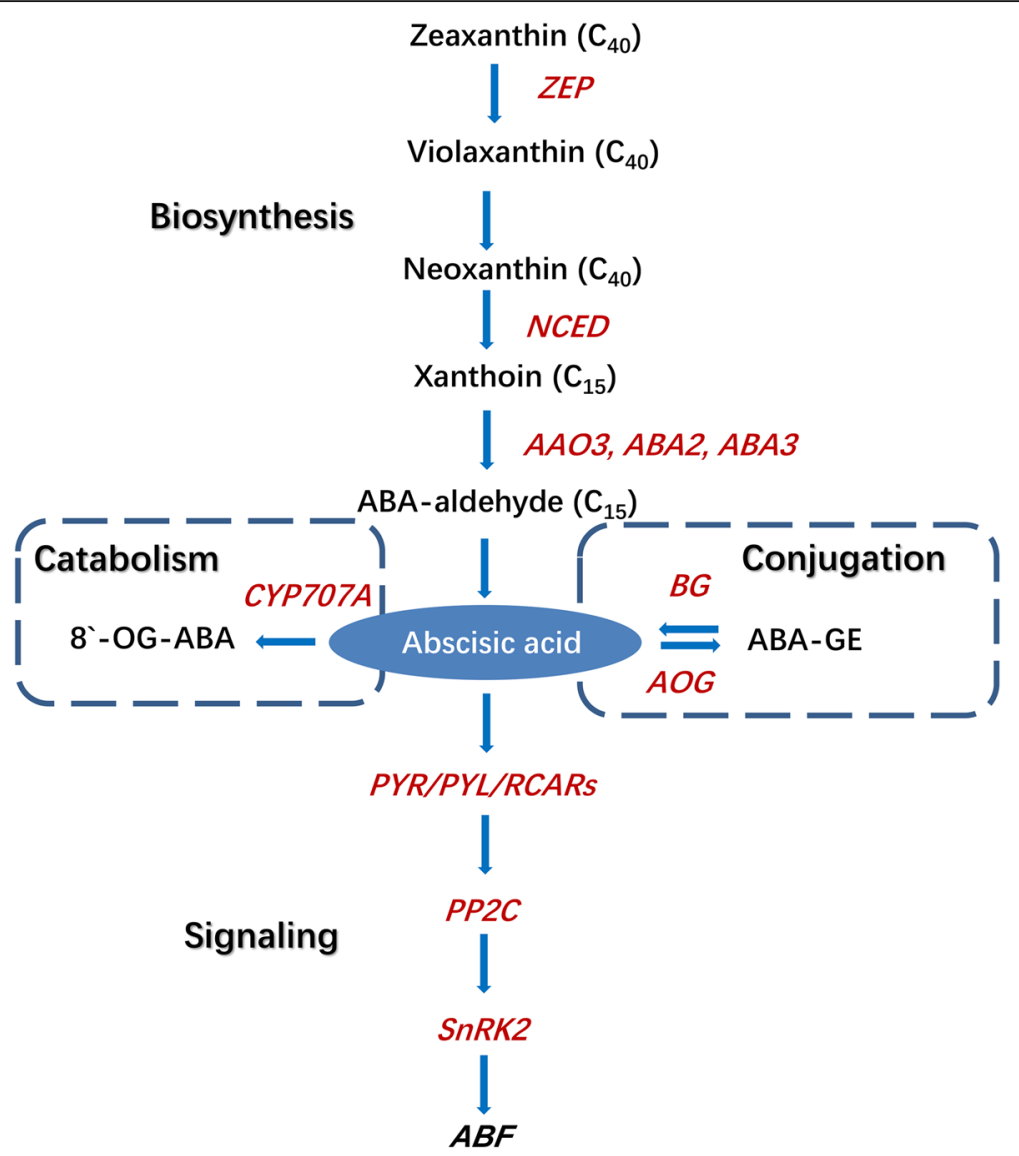

Fig. 1 ABA biosynthesis, catabolism, conjugation, and signaling pathway according to Leng et al. (2014) [5]. The expression of genes colored in red were determined in the manuscript

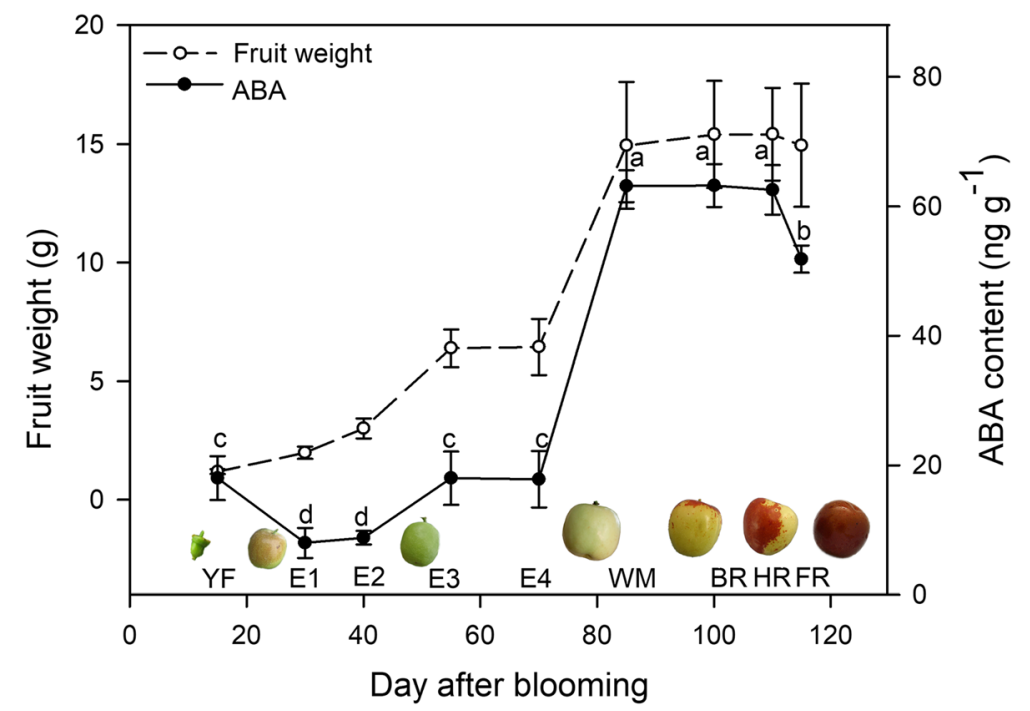

Fig. 2 Dynamic changes in fruit weight and endogenous ABA content during the 'Dongzao' fruit development and ripening process. YF, young fruit; $E$, enlarging fruit; $W M$, white mature; $B R$, beginning red; $H R$, half red; $F R$, full red. The different letters over the bars represent significant differences between the mean values ( $p<0.05$, Duncan test) 

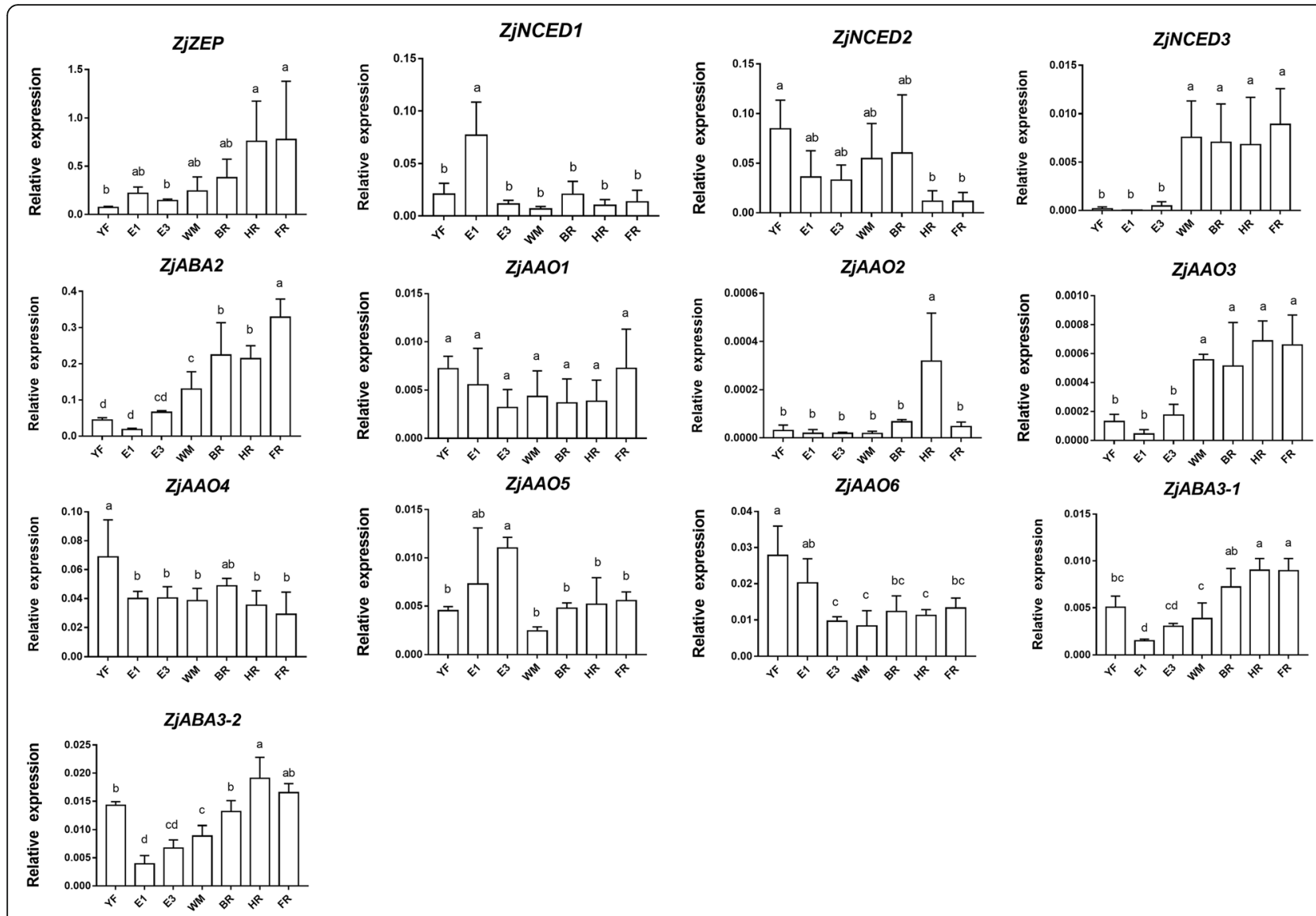

Fig. 3 Relative expression of ABA biosynthesis genes during 'Dongzao' fruit development and ripening. YF, young fruit; E, enlarging fruit; WM, white mature; BR, beginning red; HR, half red; FR, full red. The different letters over bars represent significant differences between the mean values $(p<0.05$, Duncan test)

expression levels of these genes were low in immature fruit but then increased after the WM stage. ZjBG1 showed higher expression in the YF and E1 stages than in the other stages, but the expression then decreased and did not significantly change. ZjBG2 showed higher expression at the HR, FR and YF stages than at the other stages. $Z j B G 6$ showed relatively high expression at YF and did not significantly change in other stages. $Z j B G 7$ showed higher expression in the YF, WM, and BR stages than in the other stages. In addition, we also identified two ABA-glucosyltransferases (AOG), but their expression levels were extremely low and were not shown in the figure.

\section{Relative expression of $A B A$ signaling pathway genes} during Chinese jujube fruit development and ripening With respect to the signaling pathway, 6 genes encoding ABA receptors as well as $P Y R / P Y L / R C A R$ were identified. ZjPYR1 and ZjPYR5 showed relatively high expression in the YF and E1 stages, but their expressions were very low after the onset of ripening (Fig. 5a). The expression levels of $Z j P Y L 8$ and $Z j P Y L 9$ were low in immature fruit but slightly increased after WM. In addition, ZjPYL2 and ZjPYL4 were not expressed in jujube fruit.

Among the eight identified PP2Cs, ZjPP2C4, ZjPP2C5, $Z j P P 2 C 6, Z j P P 2 C 7$, and ZjPP2C8 had similar expression patterns along with $A B A$ accumulation, with rapidly increased expression at the WM stage (Fig. 5b). However, ZjPP2C1 showed lower expression in the YF and E1 stages but higher expression in HR fruit. ZjPP2C2 exhibited relatively high expression in the YF stage, but its expression was consistent afterwards. The relative expression of $Z j P P 2 C 3$ also did not significantly change during the whole fruit growth process.

Seven $S n R K 2$ s were identified from the Chinese jujube genome. ZjSnRK2-3, ZjSnRK2-4 and ZjSnRK2-7 showed relatively low expression in immature fruit, but their expression significantly increased with ABA accumulation. In addition, ZjSnRK2-1 showed lower expression in the YF and E1 stages than in the other stages, but its expression increased beginning at the E3 stage, with maximum expression occurring at the WM stage (Fig. 5c). ZjSnRK22 had relatively high expression at YF, but the expression remained consistently in the other stages. ZjSnRK2-5 and 


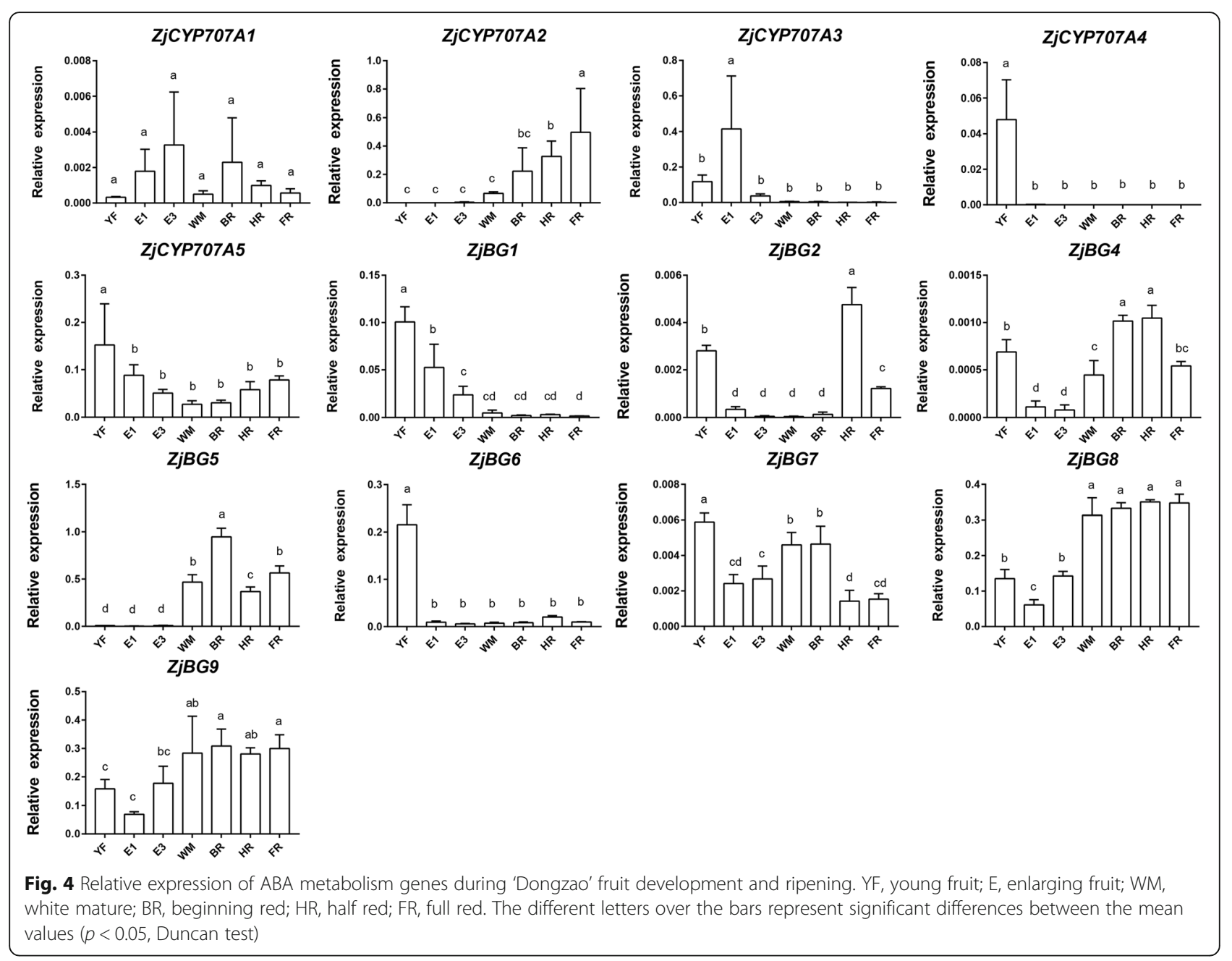

ZjSnRK2-6 showed relatively consistent expression patterns during fruit development and ripening, although ZjSnRK2-6 showed maximum expression in HR fruit.

\section{Changes in maturity and hormone levels of WM Chinese jujube fruit in response to $A B A$ and NDGA}

To explore the role of $A B A$ in regulating Chinese jujube fruit ripening, WM fruit were harvested and treated with exogenous ABA or NDGA. As expected, the endogenous ABA concentration strongly increased and remained at a high level during shelf storage in ABA-treated fruit (Fig. 6a). At 3 DAT, a moderate peak in ABA content was found in both CK- and ABA-treated fruit, although the content was slightly lower in NDGA-treated fruit at this time point. The changes in respiration rate first tended to decrease but then tended to moderately increase during storage (Fig. 6b). Exogenous ABA treatment highly increased the respiration rate at $1 \mathrm{DAT}$, but the effect was subsequently not as significant. NDGA significantly inhibited fruit respiration, as the level decreased in the treated fruit compared with the control fruit. In addition, ethylene production was very low, and ABA treatment induced a significant increase at 1 DAT, while NDGA had a limited effect on ethylene production (Fig. 6c).

\section{Identification of DEGs from comparative transcriptome analysis}

To investigate the molecular basis of ABA-associated ripening processes, the WM jujube fruit that underwent $\mathrm{ABA}$ and NDGA treatment were subjected to transcriptome sequencing at $1 \mathrm{DAT}$. In total, $41.26 \mathrm{~Gb}$ of raw sequencing data comprising $275,139,538$ reads were generated; after quality control, 271,970,678 clean reads were used for further analysis (Additional file 1). By alignment with the jujube genome, an average of $87.42 \%$ reads were uniquely mapped for each library (Additional file 2), and the relative expression levels of 31,031 transcripts were calculated. By comparative transcriptome analysis, 1103 DEGs were identified, with 456 upregulated and 647 downregulated transcripts detected in response to exogenous ABA (Fig. 7a). Moreover, 505 gene 


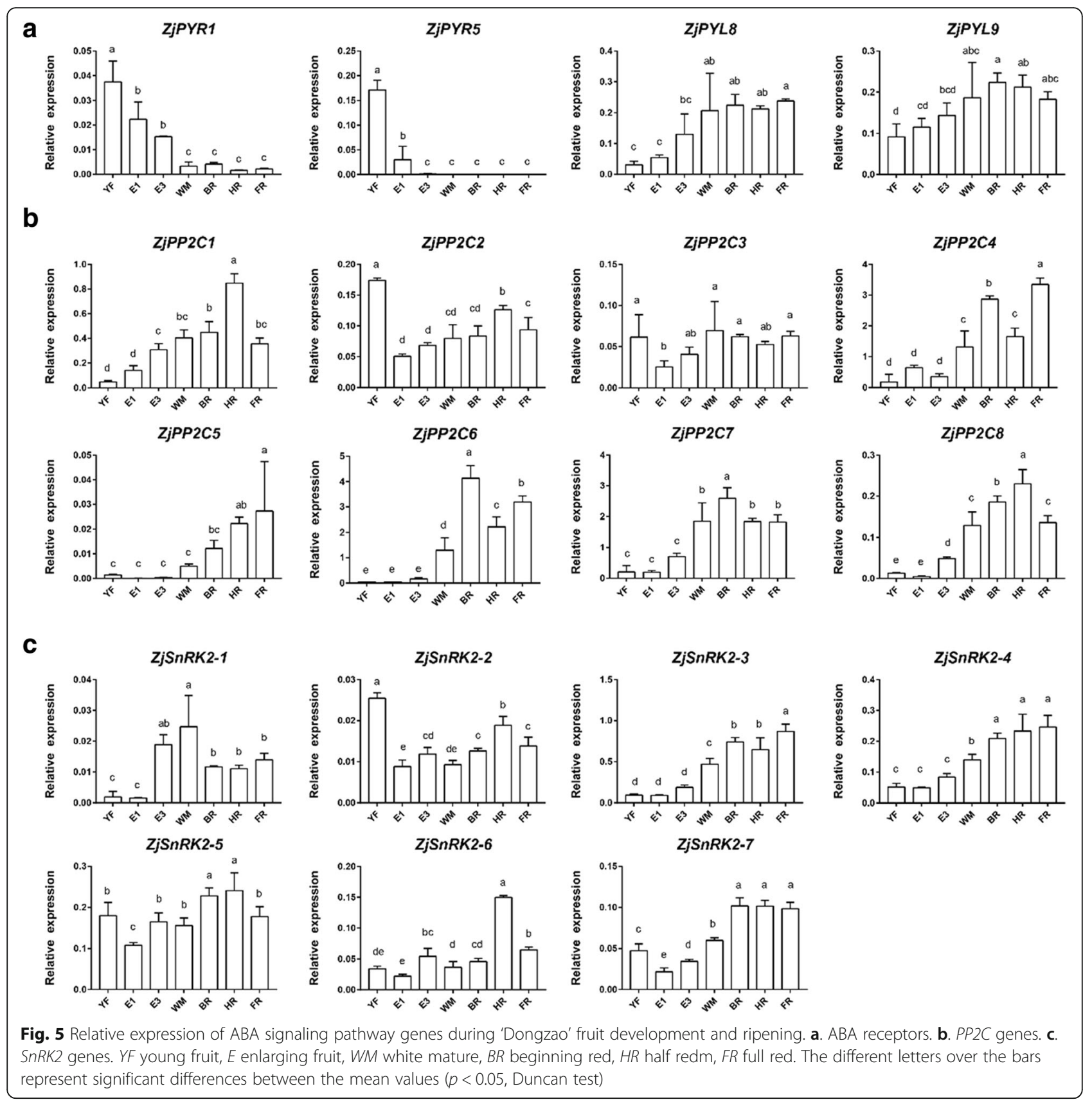

transcripts significantly changed in response to NDGA treatment, of which 45 genes were upregulated and 460 were downregulated (Fig. 7b). When these outputs were compared, 83 DEGs were simultaneously induced by both ABA and NDGA (Fig. 7c).

\section{Functional enrichment of DEGs by GO, KEGG, and MapMan analyses}

To reveal the differences in ABA and NDGA treatment effects on jujube fruit ripening, we enriched the putative function of specific DEGs using GO annotation, generating 31 and 32 classes at level 2 for the ABA (1020) and
NDGA (422) treatments, respectively (Additional file 3). The membrane, cell part, and cell classes were highly represented in the cellular component (Fig. 8a). The catalytic activity and binding classes were highly enriched in molecular function. Last, the metabolic process, single-organism process, and cellular process were involved in the most highly enriched biological processes.

The KEGG pathway analysis enriched the DEGs into 83 and 72 pathways for the ABA and NDGA treatments, respectively (Additional file 4). These pathways were mostly associated with 10 metabolic processes (Fig. 8b), 

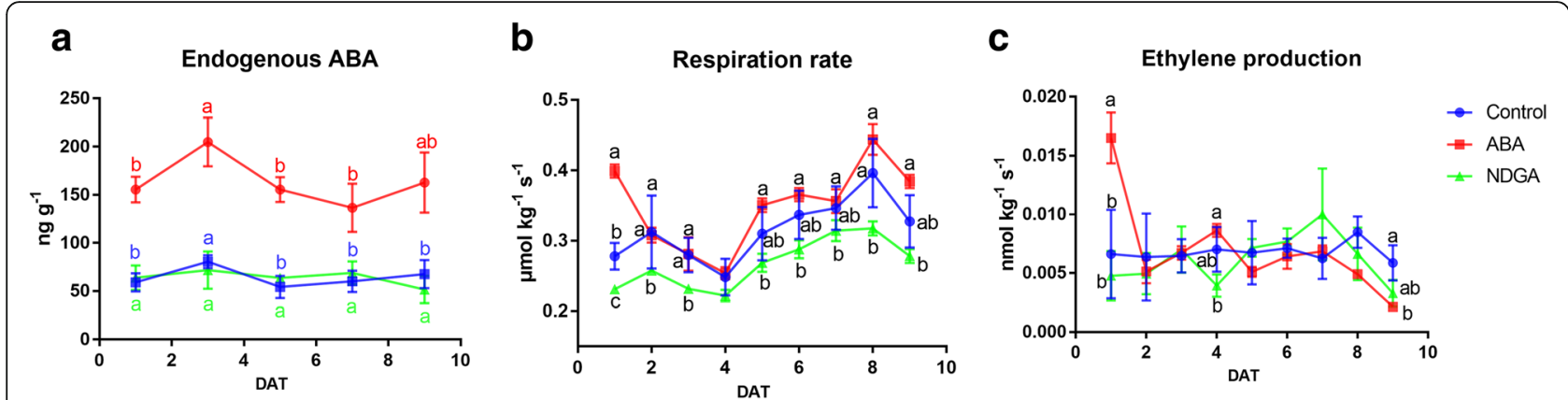

Fig. 6 Changes in hormone levels, respiration rate, and ethylene production of WM 'Dongzao' fruit in response to exogenous ABA and NDGA treatment. a. Endogenous ABA content. $\mathbf{b}$. Respiration rate. $\mathbf{c}$. Ethylene production. The different letters over the bars represent significant differences between the mean values $(p<0.05$, Duncan test)

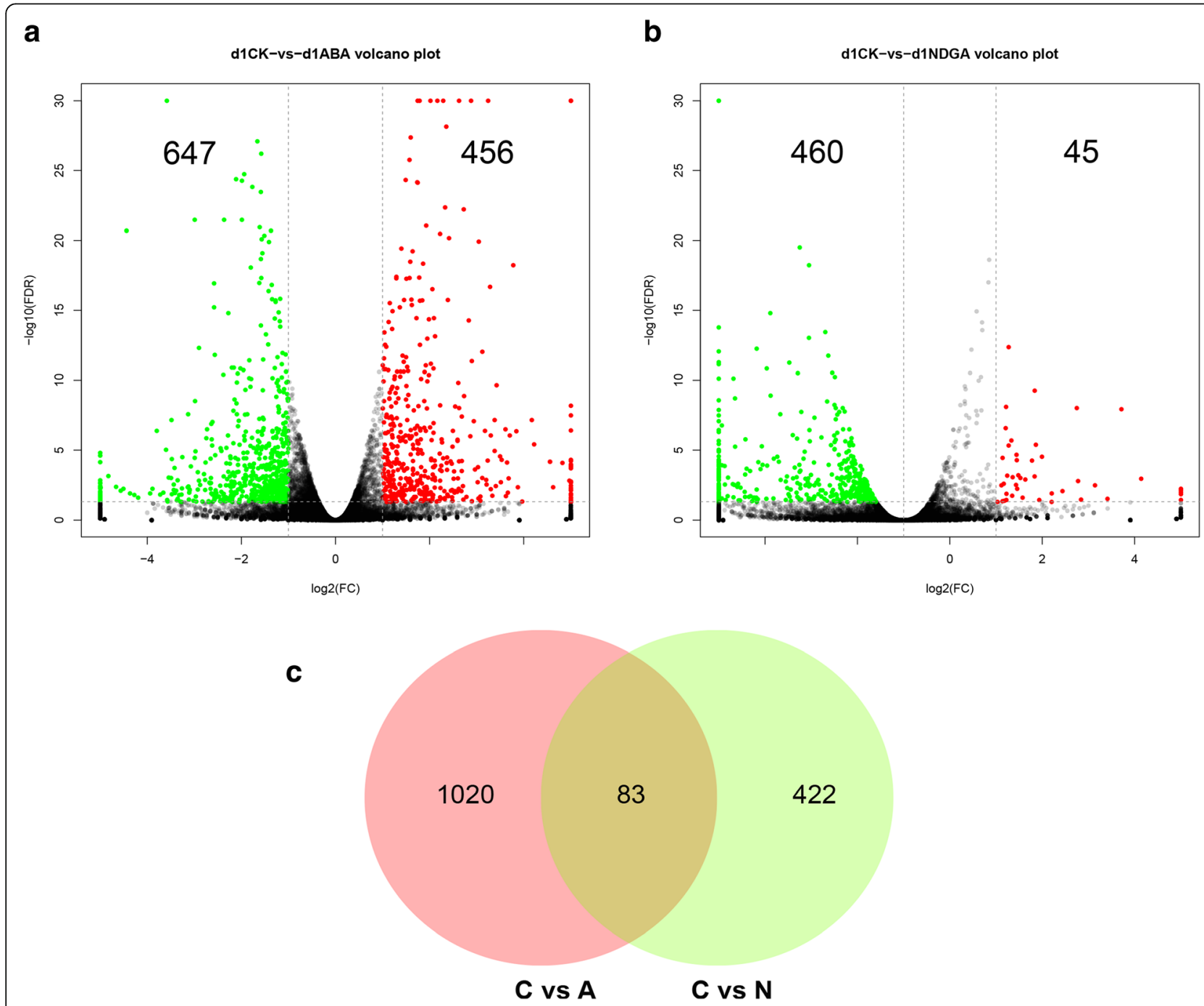

Fig. 7 Identification of DEGs in response to ABA and NDGA treatment at 1 DAT. a. Volcano plot analysis between the control (C) and ABA (A)treated fruit. The green plot indicates the downregulated transcripts, and the red plot represents the upregulated transcripts. The threshold for DEGs was set at a fold change $\geq 2$ and an FDR $\leq 0.05$. b. Volcano plot analysis between the control (C) and NDGA (N)-treated fruit. c. Venn diagram showing the number of specific and common DEGs between the ABA and NDGA treatments 


\section{a}

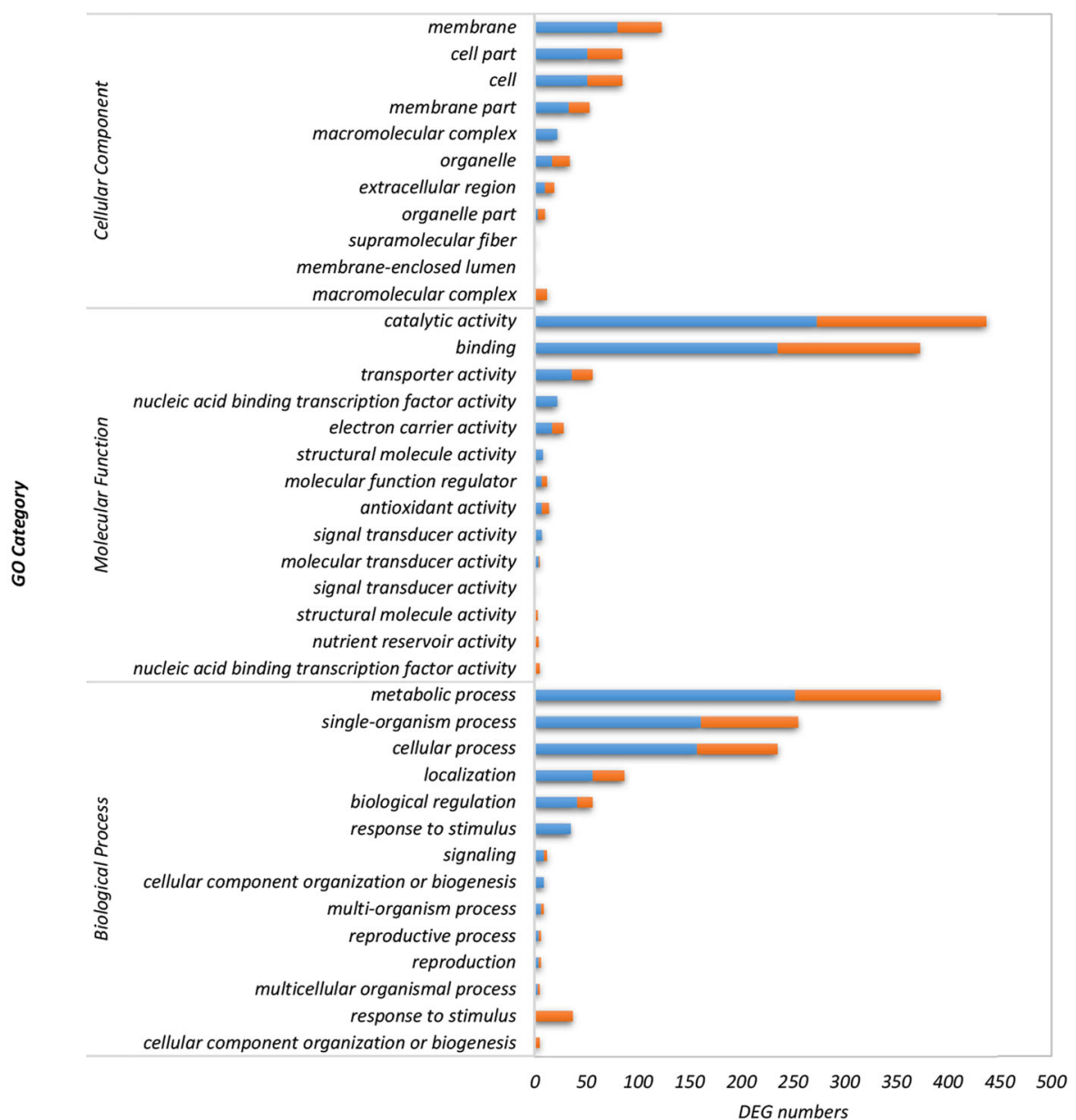

b

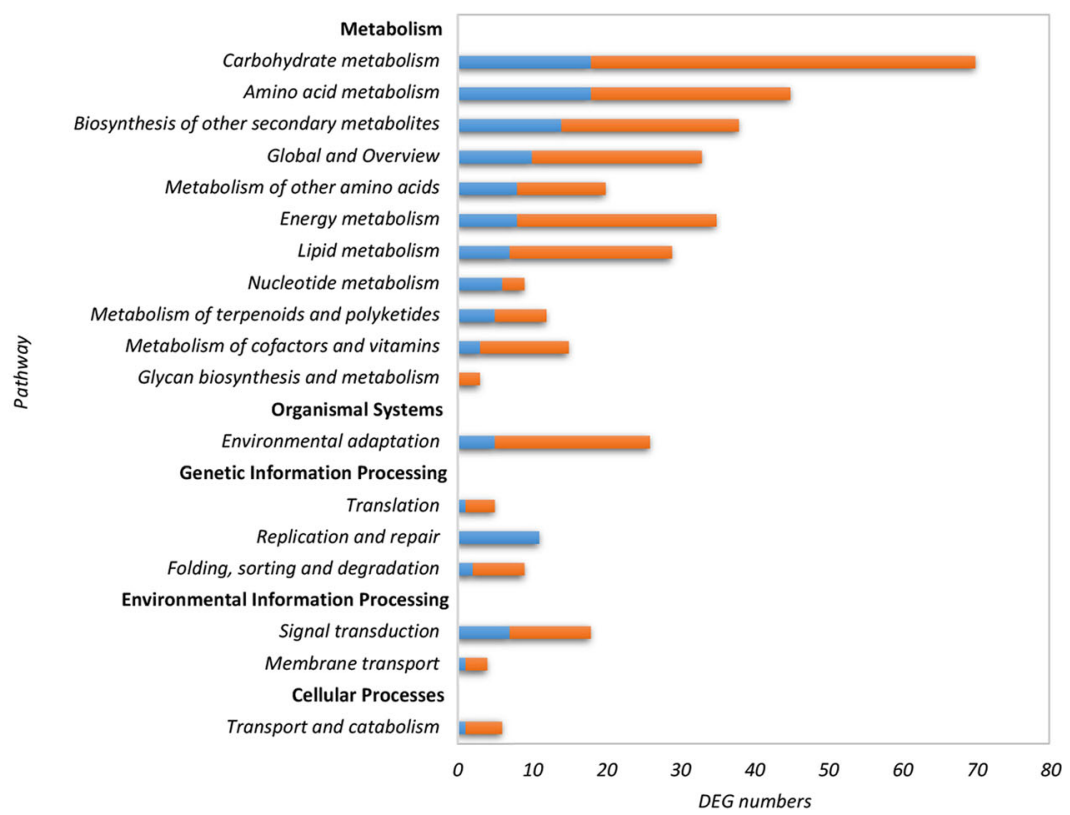

Fig. 8 Functional enrichment of DEGs induced by ABA and NDGA treatments. a. GO enrichment analysis. b. KEGG pathway analysis 
including those affecting carbohydrates, amino acids, the biosynthesis of other secondary metabolites, other amino acids, energy, lipids, nucleotides, terpenoids and polyketides, cofactors and vitamins, and glycan biosynthesis and metabolism. Moreover, genes related to environmental adaptation, translation, replication and repair, folding, sorting and degradation, signaling transduction, membrane transport, and transport and catabolism were also highly represented in pathway enrichment in response to the treatments.

In addition, MapMan analysis allowed us to gain an overview of the transcriptional changes in metabolic processes upon ABA and NDGA treatments. In response to exogenous ABA, DEGs related to photosynthesis metabolism and several secondary metabolite (terpene and flavonoid) processes were significantly suppressed, while cell wall modification, lipid metabolism, and starch and sucrose metabolism were somewhat promoted (Fig. 9a, Additional file 5). In addition, NDGA significantly inhibited these metabolic processes, which included secondary metabolism, cell wall modification, lipid metabolism, amino acid metabolism, and starch and sucrose metabolism (Fig. 9b, Additional file 5).

\section{DEGs related to plant hormone metabolism and signaling} in response to $A B A$ and NDGA treatments

A number of DEGs related to phytohormone metabolism and signaling were identified (Fig. 10, Additional file 6). In ABA metabolism, exogenous ABA downregulated the expression of ZjNCED2 (Zj.jz004177006) and upregulated the expression of two beta-glucosidases (ZjBG4, Zj.jz038707010; ZjBG5, Zj.jz044273026), while NDGA promoted the transcription of ZjCYP707A1 (Zj.jz017079277) and suppressed the transcription of ZjBG5 (Zj.jz044273026). In the signaling pathway, gene expression was not significantly induced by exogenous ABA or NDGA.

Transcripts of genes controlling ethylene biosynthesis, including 1-aminocyclopropane-1-carboxylate synthase (ACS7, Zj.jz035147028) and 1-aminocyclopropane-1-carboxylate oxidase (ACO3, Zj.jz017079235), significantly accumulated in response to ABA treatment. However, in response to NDGA, the expression of $A C O 1$ (Zj.jz022243019) was downregulated. In ethylene signaling, expression of the receptor ERS2 (Zj.jz001627227) was strengthened by $\mathrm{ABA}$, and ETR2 (Zj.jz028715030) was highly upregulated in response to NDGA. The other genes involved in signaling were barely induced by either treatment; in addition, an ethylene-insensitive 3-binding F-box (EBF, Zj.jz019851103), which modulates ethylene-insensitive 3-like (EIN3/EIL) proteins at the posttranslational level, was upregulated by ABA but not significantly by NDGA.

The expression of an ent-kaurenoic acid hydroxylase (KAO, Zj.jz041667045) involved in the early steps of GA biosynthesis was upregulated in response to $\mathrm{ABA}$, and the expression of $K A O$ (Zj.jz001403002) was downregulated by NDGA. Moreover, two GA-20-oxidases (GA20ox, Zj.jz041763136, Zj.jz037529002), which participate in the biosynthesis of active GA, were downregulated in response to exogenous ABA. For GA degradation, a GA-2-oxidase (GA2ox, Zj.jz034557218) displayed relatively high expression in ABA-treated fruit. With respect to the signaling pathway, the transcription of DELLA1 (Zj.jz004979072) was downregulated by ABA but was not significantly induced by NDGA.

The relative expression of genes involved in auxin metabolism and signaling was barely induced by exogenous $\mathrm{ABA}$; however, a tryptophan aminotransferase (TAA, Zj.jz044581006) gene involved in the tryptophandependent pathway for auxin biosynthesis and a gretchen hagen 3 (GH3, Zj.jz043973001) gene involved in conjugation were simultaneously downregulated by NDGA treatment. In addition, exogenous ABA downregulated the expression of two genes controlling cytokinin biosynthesis, including CYP735A (Zj.jz043523077), which encodes a cytokinin trans-hydroxylase, and LOG (Zj.jz008869058), which encodes a cytokinin riboside 5'-monophosphate phosphoribohydrolase. In signaling, only a histidine-containing phosphotransferase $(A H P)$ was downregulated by ABA, and NDGA showed a limited effect on the expression levels of these genes.

\section{DEGs related to TFs in response to $A B A$ and NDGA treatments}

We also identified several TFs that were differentially expressed in response to ABA and NDGA treatments (Fig. 11, Additional file 7). Exogenous ABA upregulated the expression of 3 APETALA2/ethylene-responsive elements (AP2/ERF, Zj.jz042635005, Zj.jz044705012, and Zj.jz044537132), two zinc finger proteins (Zj.jz028519044 and Zj.jz006119044), 1 NAC domain-containing protein (Zj.jz007373078), and 1 WRKY gene (Zj.jz015029046). In addition, $A B A$ downregulated the expression of 2 AP2/ERFs (Zj.jz044531026 and Zj.jz034557065), 2 bHLHs (Zj.jz042793020 and Zj.jz042921104), 2 bZIPs (Zj.jz0137047 and Zj.jz037039133), 1 zinc finger protein (Zj.jz041823042), 3 homeobox $(H B)$ transcription factors (Zj.jz029235034, Zj.jz039989051, and Zj.jz043799010), 1 heat-shock TF (HSF, Zj.jz004979138), 6 MYBs (Zj.jz004979253， Zj.jz005919060， Zj.jz008869110, Zj.jz019661008, Zj.jz041121020, Zj.jz043819011, and Zj.jz044713001), and 2 WRKYs (Zj.jz034949026 and Zj.jz042571063).

The NDGA also induced the expression of several DEGs related to TFs, including 2 AP2/ERFs (ZjERF52, Zj.jz034557065 and Zj.jz001627224), 1 bHLH (Zj.jz031003007), 1 zinc figure protein (Zj.jz017079223), and 1 MYB (Zj.jz044713001). In 


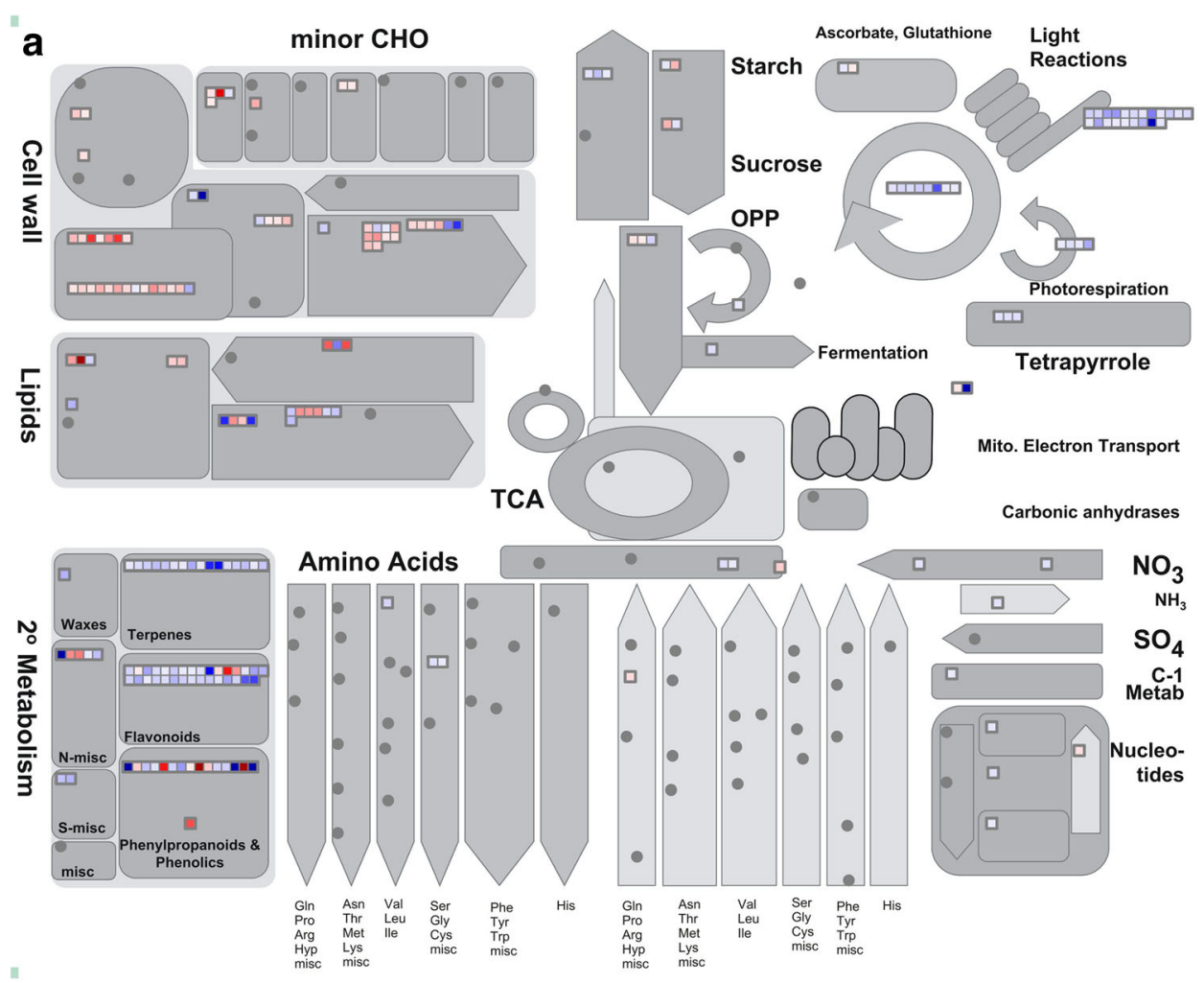

b minor $\mathrm{CHO}$
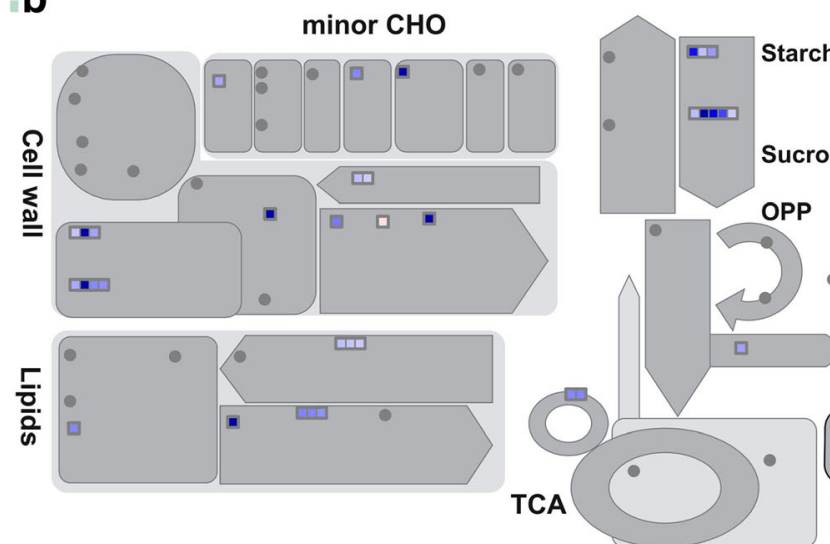

Ascorbate, Glutathione Light
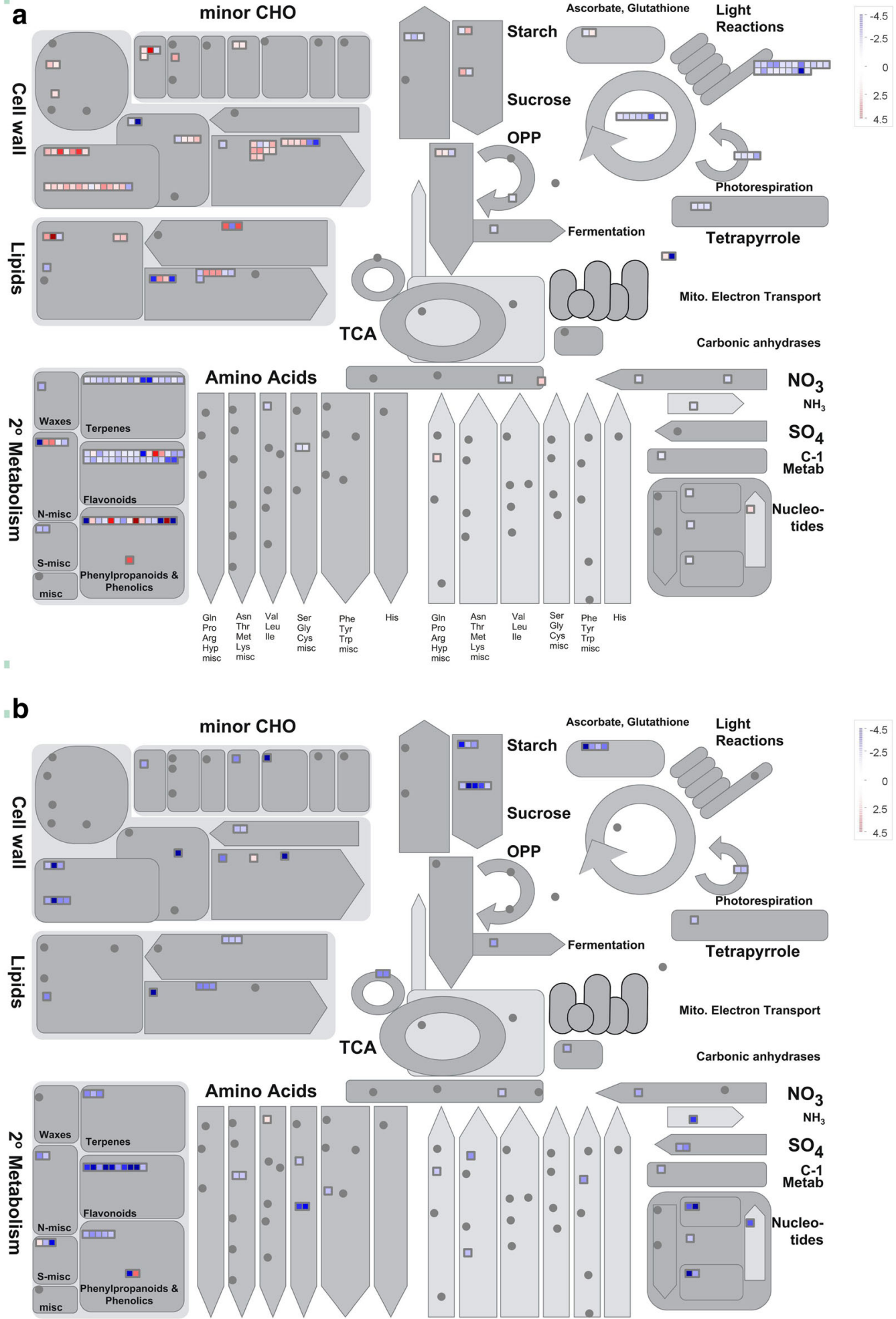

$\begin{array}{lllll}\text { GIn } & \text { Asn } & \text { Val } & \text { Ser } & \text { Phe } \\ \text { Pro } & \text { Thr } & \text { Leu } & \text { Gly } & \text { Tyr } \\ \text { Arg } & \text { Met } & \text { Ile } & \text { Cys } & \text { Trp } \\ \text { Hyp } & \text { Lys } & \text { Misc } & \text { misc } \\ \text { misc } & \text { misc } & & \text { misc } & \\ & & & \end{array}$

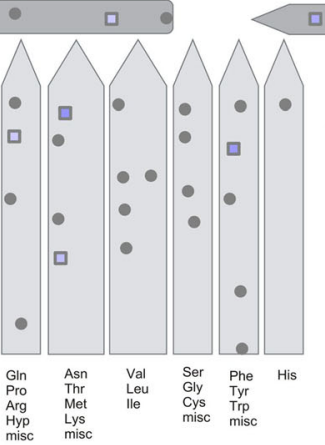

Carbonic anhydrases

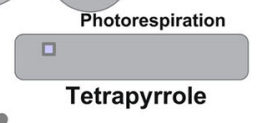

Fermentation
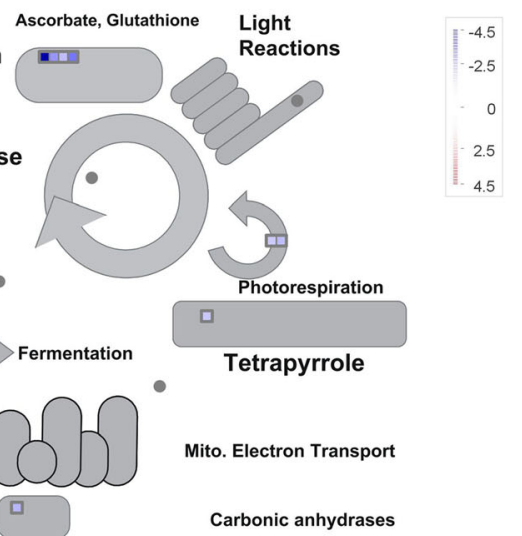

Fig. 9 Overview of DEGs involved in metabolic processes according to MapMan ontology. a. DEGs induced by ABA treatment. b. DEGs induced by NDGA treatment. The blue squares indicate downregulation, and the red squares represent upregulation. The scale bar is shown as the $\log _{2}$ (fold change) value 


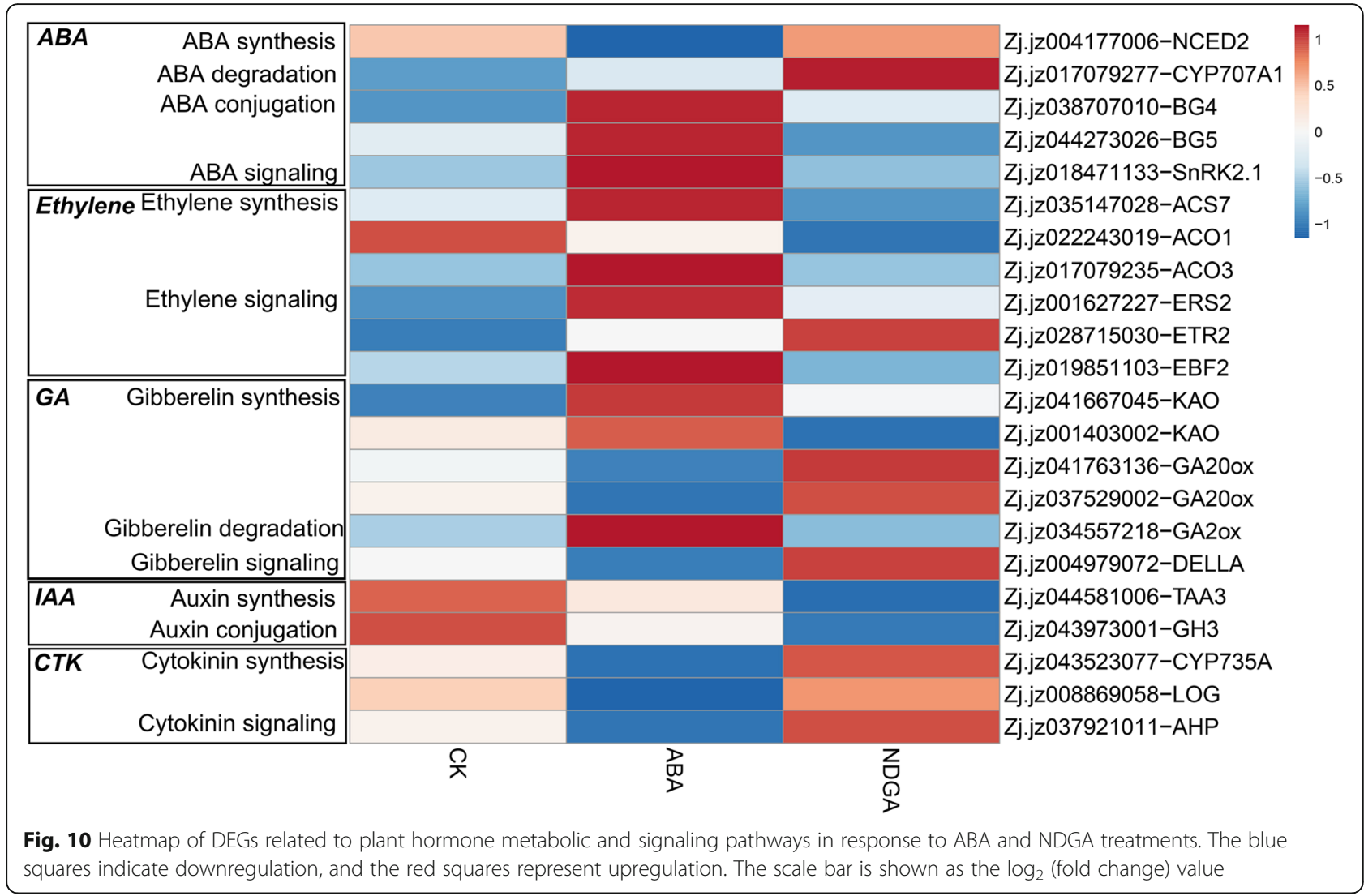

addition, the downregulated genes included $2 \mathrm{bHLHs}$ (Zj.jz015029086 and Zj.jz016733025), 5 zinc finger proteins (Zj.jz038707050， Zj.jz042849020， Zj.jz038181028, Zj.jz037267016, and Zj.jz035687008), 1 HB (Zj.jz006473038), and 2 MYBs (Zj.jz022481098 and Zj.jz014311034).

\section{Validation of transcriptomic expression levels}

Seventeen genes were selected to validate the transcriptomic expression levels by qPCR. The correlation coefficient between the RNA-seq data and relative expression ranged from 0.838-1.0 (Additional file 8), thereby confirming the reliability of the RNA-seq data.

\section{Discussion}

\section{Dynamic accumulation of endogenous ABA associated} with Chinese jujube fruit ripening

The dynamic accumulation of endogenous ABA during jujube fruit development and ripening was investigated in our study. Several studies have suggested that endogenous ABA is synthesized from pulp tissues and not transported from seeds $[8,11,23]$. The initial relatively high content of endogenous ABA at the YF stage in jujube could induce the uptake of assimilation products and the accumulation of sucrose $[8,9]$, while the rapid increase at the WM stage in response to the onset of ripening correlated with pigment accumulation, sugar metabolism, and cell wall degradation in the fruit [24]. The increase in ABA has also been reported in both climacteric and non-climacteric fruit, such as those of tomato [5], peach [25], persimmon [26], grape [24], strawberry [27], and watermelon [11]. ABA is important in inducing the onset of jujube fruit ripening because of the limited emission of ethylene at the WM stage of 'Dongzao' fruit reported in our last study [7]. Ethylene production slightly increased in the FR fruit, and ABA decreased simultaneously. We proposed that a complementary mechanism may exist, as SINCED1 RNAi tomato plants contained lower contents of ABA but presented a higher production of ethylene [28]. Nevertheless, these findings suggest a role for $\mathrm{ABA}$ in regulating the onset of jujube fruit ripening and maintaining ripening processes.

The expression of ABA biosynthesis and metabolic pathway genes during jujube fruit development and ripening suggested a balancing role for regulating the expression of NCED, CYP707A, and BG genes and determining the accumulation of endogenous ABA $[5,29,30]$. Although the ZjNCED3 expression level was not high, it tended to increase, which correlated with $A B A$ accumulation; the ZjNCED3 protein also showed an amino acid sequence similarity of 64\% with the AtNCED6 (AT3G24220) protein, which was crucial in controlling ABA biosynthesis 


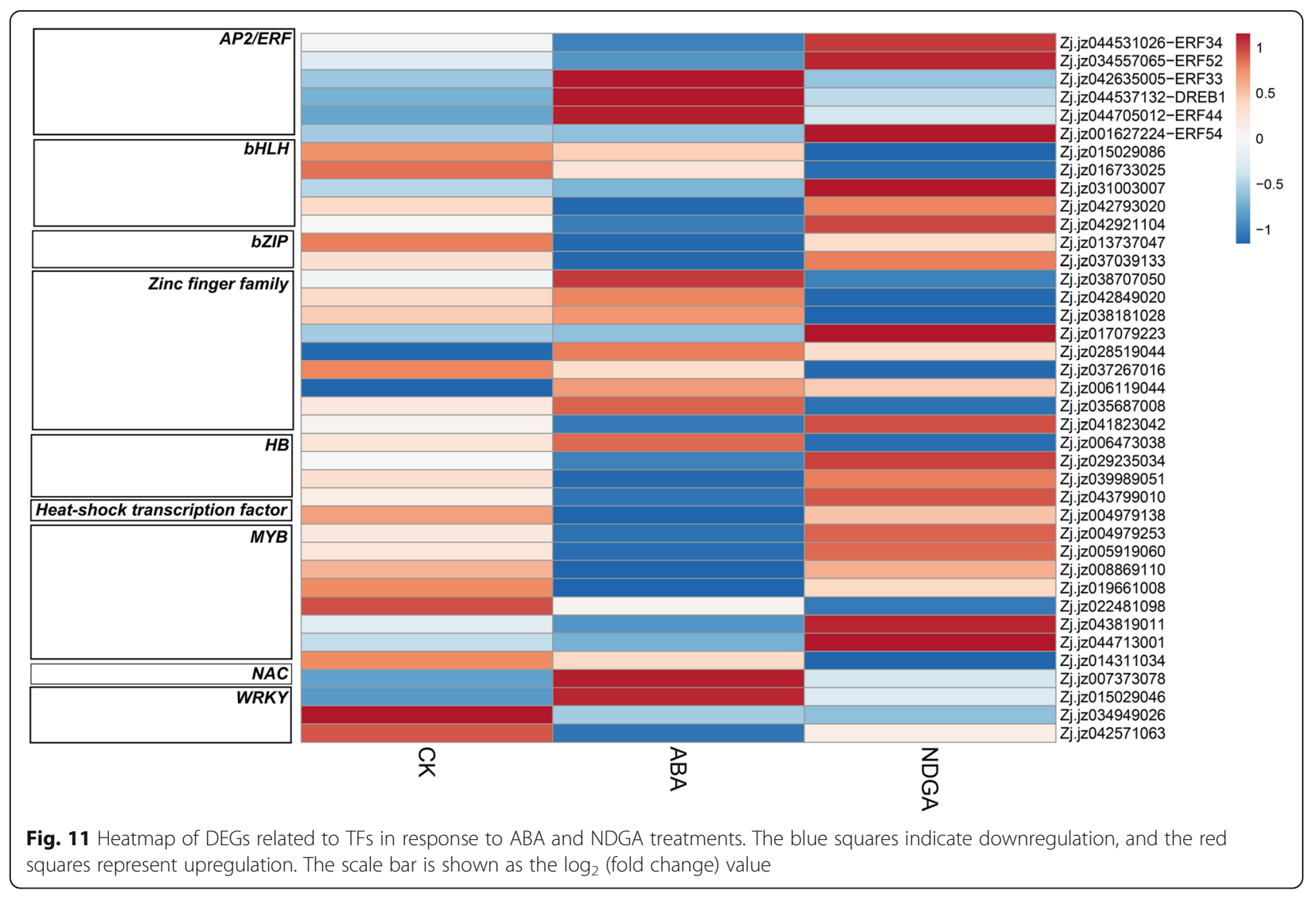

in Arabidopsis. These results indicated ZjNCED3 could also be important in determining ABA biosynthesis in Chinese jujube fruit. Although the expression of ZjNCED1 and ZjNCED2 failed to parallel the ABA accumulation, these genes may also participate in this process; for example, CSNCED2 appeared to play a subsidiary role in ABA accumulation, as the gene product was restricted to chromoplast-containing tissue in Citrus fruit [31]. While CYP707As were shown to catalyze ABA degradation [32, 33], we found that the expression of ZjCYP707A3, ZjCYP707A4, and ZjCYP707A5 decreased at the onset of jujube fruit ripening, suggesting a developmental stage-dependent expression pattern [27], which was also reported in watermelon (Cla020637 and Cla016011) [11] and mulberry (MaCYP707-1-4) [34]. In addition, $\mathrm{ZjCYP707A2}$ showed increased expression at the FR stage, which we propose is related to the decrease in ABA content at that stage. With respect to $B G$ s, the encoded enzymes catalyze the hydrolysis of glycosylated-ABA and rapidly increase the content of free $A B A$ in plant tissues [35]. Our results suggested that $Z j B G 4, Z j B G 5, Z j B G 8$, and $Z j B G 9$ are potentially involved in regulating $A B A$ accumulation due to their expression patterns being consistent with dynamic changes in $\mathrm{ABA}[5,23]$; inhibiting the expression of
FaBG3 decreased the ABA content and delayed strawberry fruit ripening [36]. In cucumber, two $B G$ s were also identified as being involved in the regulation of ABA accumulation [23].

The expression of several other genes involved in the ABA biosynthesis pathway was also determined in our study, although there are few reports related to their expression during fruit ripening. ZjZEP was consistently expressed during jujube fruit development and ripening, and its expression was also not related to ABA accumulation in Vaccinium myrtillus fruit [37]. ZjABA2 showed increased expression following dynamic changes in $A B A$, while $A t A B A 2$-overexpressing plants also presented relatively high ABA accumulation [38]; however, correlations were not detected during fruit ripening of Vaccinium myrtillus [37]. In jujube, only ZjAAO3 showed an expression consistent with dynamic changes in ABA; AAOs belong to a small gene family whose members have been purported to be functionally redundant, and only AtAAO3 participates in ABA biosynthesis in Arabidopsis [39, 40]. In addition, the expression of ZjABA3-1 conformed to that of $Z j A A O 3$; $A B A 3$ encodes a molybdenum cofactor sulfur enzyme and functions together with $A A O 3$ [30]. A previous study also showed that expression of $A t A B A 3$ was positively correlated with the ABA content in Arabidopsis [41]. 
Transcription of ABA signaling pathway genes during Chinese jujube fruit development and ripening

The ABA signaling pathway, consisting of $P Y R / P Y L /$ $R C A R-P P 2 C-S n R K 2$, has been widely reported in plants [5]. In our study, ZjPYR1 and ZjPYL5 were expressed at a relatively high level in immature fruit, but their expression was low after the onset of ripening, while ZjPYL8 and ZjPYL9 showed the opposite trend, with relatively high expression during ripening. According to the results of a phylogenetic analysis (Additional file 9), ZjPYR1 belonged to clade III, and ZjPYL5 belonged to clade II. These two clades also showed a negative correlation between their expression and ABA accumulation in Citrus fruit (CsPYR1, CsPYL4, and CsPYL5) [42]. ZjPYL8 and ZjPYL9 belonged to clade I, and the genes involved in this clade were found to have positively accumulated along with the increase in ABA, as did the CsPYL8 and CsPYL9 genes in Citrus [42]; SIPYL1, SIPYL2, and SIPYL3 in tomato [43]; and FaPYR1 in strawberry [44]. Therefore, our findings suggest that ZjPYR1 and $Z j P Y L 5$ are key genes for ABA signaling in immature jujube fruit, while $Z j P Y L 8$ and $Z j P Y L 9$ are more important for maintaining signaling during fruit ripening processes.

PP2C proteins act as negative regulators in ABA signaling [45], and they can combine with receptors and form into components of protein kinase complexes, which regulate fruit sensitivity to ABA signaling [42, 46, 47]. Our study identified eight genes belonging to the $P P 2 C$-A subfamily, and five of them (ZjPP2C4-8) were upregulated along with ABA accumulation. Several previous studies also showed that the expression of partial PP2Cs accumulated during fruit ripening in Citrus (CsABI1, CsHAB1, CsAHG1, CsAHG3, and CsHAI3), tomato (SlPP2C1 and $S l P P 2 C 5)$, and grape ( $V v P P 2 C 3, V v P P 2 C 6, V v P P 2 C 7$, and $V v P P 2 C 9)$ [42, 43, 48]. However, more evidence is still needed to elucidate the relationships between $P P 2 C$ s and sensitivity to ABA signaling.

Additionally, seven $\operatorname{SnRK2\text {s}}$ were identified in our study and were classified into three clades according to studies in both Citrus and tomato [42, 43]. However, only clade III was suggested to be involved in the ABA signaling pathway due to the enriched Asp (D) residues, which were located within the C-terminal sequences and were known as kinase binding sites [49]. Our study found that only ZjSnRK2-2 and ZjSnRK2-5 belonged to this clade (Additional file 9), and they did not show any significant correlations between their transcripts and ABA content. This phenomenon was consistent with previous findings in Citrus, tomato, and apple fruit [42, 43, 50]. For clades I and II, ZjSnRK2-3, ZjSnRK2-4, and ZjSnRK2-7 also accumulated with the increase in ABA content, which suggests that these genes participate in ripening regulation but are not involved in ABA signaling [51]. Nevertheless, ZjSnRK2-2 and ZjSnRK2-5 are relatively important for maintaining ABA signaling in jujube fruit.

\section{$A B A$ acts as a positive regulator in Chinese jujube fruit ripening}

To further investigate the role of $\mathrm{ABA}$ in modulating jujube fruit ripening, WM 'Dongzao' fruit were treated with a low concentration of ABA or NDGA. Our results showed that $A B A$ treatment promoted a rapid accumulation of endogenous $\mathrm{ABA}$ and partly increased fruit respiration and ethylene production. These results were similar to those of a recent study showing that $50 \mathrm{mg} \mathrm{L}^{-1} \mathrm{ABA}$ promoted WM 'Dongzao' fruit ripening and reduced fruit quality during postharvest storage [52]. In addition, our research showed that $50 \mathrm{mgL}^{-1}$ NDGA inhibited the increase in endogenous ABA at 3 DAT, significantly decreased fruit respiration, but slightly affected ethylene production in our study. NDGA is an inhibitor of ABA biosynthesis because it could interact with NCED enzymes with regard to its permeation speed [53]. Therefore, NDGA reduced ABA biosynthesis but did not completely suppress the process $[54,55]$. Thus, these results suggested that $\mathrm{ABA}$ acted as a potential positive regulator in jujube fruit ripening.

The comparative transcriptome analysis provided an overview of DEGs correlated with altered metabolic processes in response to the treatments. Previous studies have suggested that the transition underlying fruit ripening induced by $\mathrm{ABA}$ is associated with the suppression of vegetative growth metabolism and the activation of ripening-specific pathways $[14,56]$. In our study, genes related to photosynthetic metabolism and several secondary metabolites were found to be somewhat downregulated by ABA (Additional file 5). Photosynthetic metabolism is regarded as a switch that induces the transition to fruit ripening via downregulated gene expression in response to exogenous ABA [13, 14, 57]. Moreover, genes involved in the metabolism of flavones, flavanols and flavonoids were downregulated by ABA at 1 DAT, suggesting a possible stress response of jujube fruit to exogenous ABA. In grape, the expression of genes associated with phenylpropanoid biosynthesis and flavonoid biosynthesis was also downregulated by ABA at $20 \mathrm{~h}$ posttreatment, but the expression was upregulated at $44 \mathrm{~h} \mathrm{[14]}$. We speculated that secondary metabolism would be positively induced by ABA after 1 DAT because of its positive role in regulating secondary metabolites; this process is common to tomato, grape and strawberry $[13,16,58]$.

On the other hand, we found that genes involved in lipids, cell wall modification, starch, and sucrose metabolism were somewhat positively induced by ABA and NDGA, suggesting a correlation between $\mathrm{ABA}$ and ripening-related metabolism. In lipid pathways, altered gene expression participates in the modification of the cell membrane, the biosynthesis of aromatic compounds, and lignification induced by wounding $[12,19,59,60]$. With respect to cell 
wall modification, genes related to cellulose synthesis, cell wall proteins, and cell wall degradation and modification were somewhat upregulated by $\mathrm{ABA}$, indicating that $\mathrm{ABA}$ has a potential role in regulating changes in fruit texture $[5,17,61]$. In the starch biosynthesis pathway, genes encoding ADP-glucose pyrophosphorylase and isoamylase were downregulated by ABA, while NDGA downregulated the expression of genes controlling starch degradation, including two alpha-amylase- and a beta-amylase-encoding genes. These results suggested that ABA was involved in starch metabolism, just as ZmEREB156 positively modulated starch biosynthesis via the synergistic effect of sucrose and ABA in maize [62]. In sucrose metabolism, genes encoding the vacuolar invertase, hexokinase, and sugar transporters were significantly induced by $\mathrm{ABA}$, indicating that $\mathrm{ABA}$ promoted sucrose degradation but maintained the content of glucose and fructose, while the expression of these genes was inhibited after NDGA treatment. These results suggest a putative role of ABA in regulating sucrose metabolism, and genes related to sucrose metabolism were also found to be activated after $44 \mathrm{~h}$ in grape fruit treated with exogenous ABA [14]. Overall, our study provided comprehensive insight into the role of $\mathrm{ABA}$ in regulating jujube fruit ripening-related metabolism, and additional studies are still needed to elucidate the complex regulatory mechanism involved.

\section{The regulatory network of $A B A$ in modulating Chinese jujube fruit ripening}

The crosstalk between ABA and other plant hormones and TFs was analyzed in our study via transcriptomic data (Fig. 12). Exogenous ABA promoted a rapid accumulation of endogenous $A B A$ in treated fruit but inhibited the expression of $Z j N C E D 2$, indicating a negative feedback regulation in response to exogenous $A B A$, as was the case for CsNCED1 in Citrus and LeNCED2 in tomato [20, 54]. ZjBG4 and ZjBG5 were upregulated in response to $A B A$, and these two genes showed a positive response to $\mathrm{ABA}$ accumulation during fruit ripening, which is crucial in regulating the ABA content $[11,23]$. Conversely, NDGA treatment promoted the expression of ZjCYP707A1 but inhibited ZjBG5, thereby inhibiting the increase in ABA content at 3 DAT. These results corroborated the role of transcriptional balance among $N C E D, C Y P 707 A$, and $B G$ in maintaining ABA accumulations $[11,19]$.

We also found that genes involved in ethylene biosynthesis and perception, including ZjACS7, ZjACO3, and ZjERS2, were upregulated by ABA; similar results were also found in tomato (LeACS2, LeACS4, and LeACO1) and grape $(V v A C O 1)$, suggesting that ABA may act as an upstream regulator of the ethylene pathway $[8,25,55]$. With respect to the ethylene signaling pathway, ZjERS2 was upregulated by ABA. The increased expression responds to the degradation of receptors induced by the binding with ethylene $[63,64]$. The genes involved in the signaling pathway were rarely induced by the treatments, although the expression of the $Z j E B F 2$ gene was upregulated by $\mathrm{ABA}$. Moreover, $E B F$ regulates the expression of ethylene-insensitive 3-like (EIL) at the posttranscriptional level $[65,66]$; therefore, this result also indicated an indirect regulation of the ethylene signaling pathway by ABA.

In our study, the expression of several genes involved in the biosynthesis, metabolism, and signaling of GA, auxin, and cytokinin; GA20ox, CYP735A, and LOG, which are involved in the biosynthesis of GA and cytokinin; a DELLA protein involved in GA signaling, and an $A H P$ gene involved in cytokinin signaling was downregulated in response to exogenous $\mathrm{ABA}$. ABA also upregulated the expression of GA2ox, which regulates the degradation of GA. In addition, NDGA inhibited the expression of genes (TAA and GH3) involved in auxin biosynthesis. There is evidence suggesting that GA, auxin, and cytokinin decrease during fruit ripening in strawberry, grape, and tomato [67-69]. Moreover, litchi, strawberry, and sweet cherry fruit treated with these phytohormones inhibit or delay fruit ripening processes $[15,19,22,70]$. Therefore, ABA would crosstalk with hormones which negatively correlated with ripening.

With respect to the TFs induced by ABA and NDGA, DEGs related to the AP2/ERF, NAC, WRKY, bHLH, bZIP, $H B, M Y B$, zinc finger protein, and $H s f$ TFs were identified. These TFs are extensively involved in fruit metabolism such as phytohormone metabolism and signaling, the accumulation of flavonoids and anthocyanins, and the response to stress [6,71]. Exogenous ABA inhibited the expression of ZjERF34 and ZjERF52, which were previously suggested to be downregulated during ripening in our last study, while ZjDREB1 was upregulated by $\mathrm{ABA}$ and was also found to be positively correlated with ripening [72]. In addition, two bZIPs were downregulated by ABA, and Zj.jz037039133 is probably located at the end of $\mathrm{ABA}$ signaling as an $\mathrm{ABA}$ response factor; this factor was suggested to be involved in the regulation of $\mathrm{ABA}$ and phenol accumulation in grape [73]. Our study also identified 6 MYB TFs that were downregulated by $\mathrm{ABA}$ and a TF that has an extensive role in modulating fruit coloration and texture [74]. A number of zinc finger proteins were found to be induced by exogenous treatment, and C2HC-type zinc finger proteins could respond to the resistance of oxidative stress induced by exogenous ABA [75]. We also found that the $N A C$ gene was upregulated by $\mathrm{ABA}$ and was found to be involved in the regulation of ethylene signaling, fruit pigmentation and fruit softening [76]. Overall, the identification of these TFs provided us with broad insight 


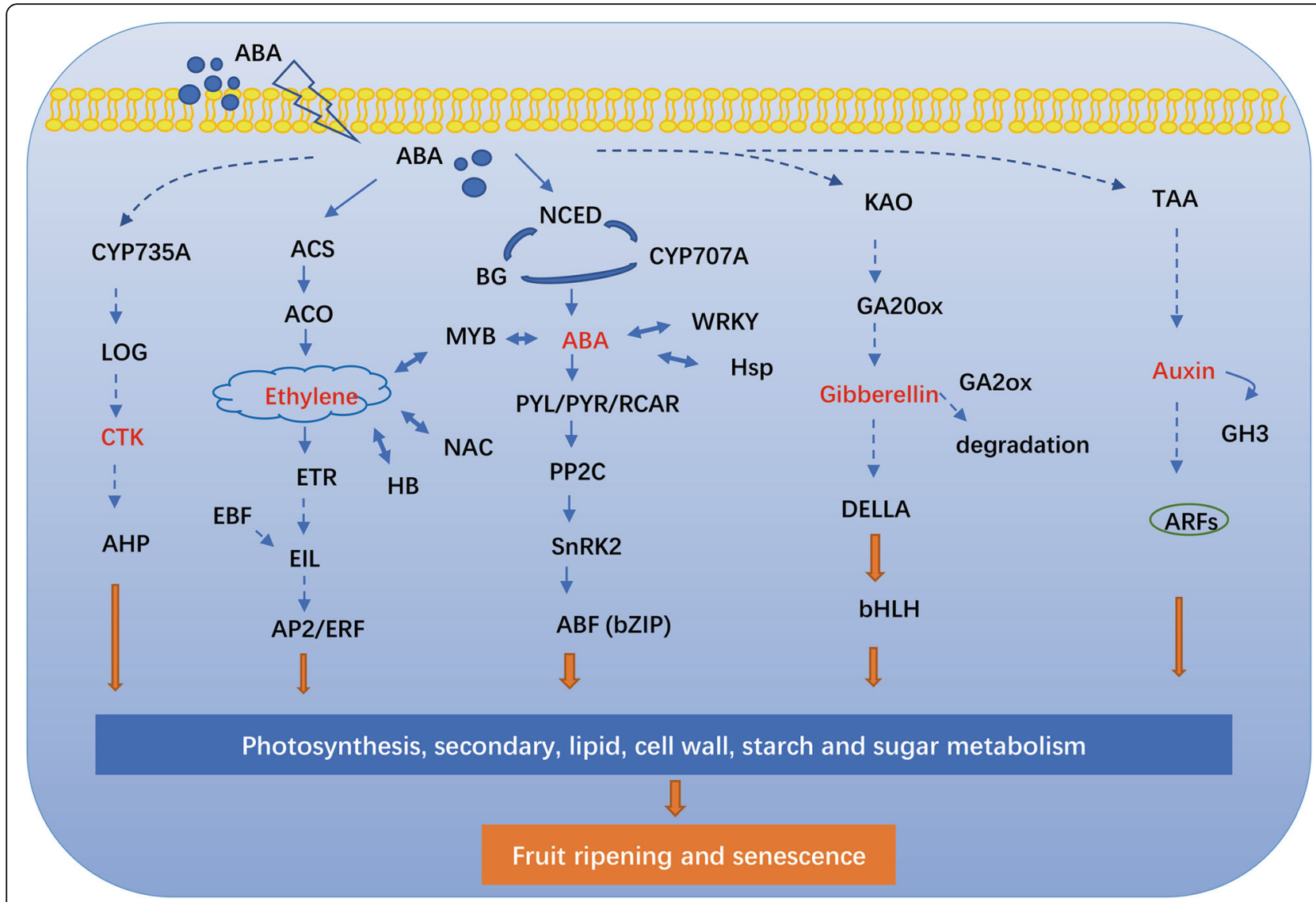

Fig. 12 Putative regulatory network for Chinese jujube fruit ripening

into the complex regulation of $\mathrm{ABA}$ involvement in modulating jujube fruit ripening, and the potential mechanism of these TFs should be preferentially studied in the future.

\section{Conclusions}

Our study provided comprehensive insights into the role of $\mathrm{ABA}$ in Chinese jujube fruit ripening and revealed a positive correlation between the onset of fruit ripening and the accumulation of endogenous $\mathrm{ABA}$, which was modulated by the interaction among the expression of NCED (ZjNCED3), CYP707A (ZjCYP707A2), and BGs (ZjBG4, ZjBG5, ZjBG8, and ZjBG9). The signaling pathway consisting of a receptor (ZjPYL8), PP2Cs (ZjPP2C4, ZjPP2C5, ZjPP2C6, ZjPP2C7, and ZjPP2C8) and SnRK2s (ZjSnRK2-2 and ZjSnRK2-5) was important in regulating fruit sensitivity to ABA. In addition, ABA had a dominant role in modulating jujube fruit ripening, and our study further elucidated ABA-associated metabolism and predicted a network of jujube fruit ripening regulations. These findings would be helpful for further improvements to strategies for jujube fruit storage and for gaining insights into understanding complex non-climacteric fruit ripening.

\section{Methods}

Plant materials, treatments and storage

Fruits of a popular Z. jujuba cultivar, 'Dongzao', were harvested from adult trees grown in our experimental orchard in Xuzhuang, Dali, Shaanxi, China (N34.52, E109.56). The sampling periods were selected according to days after full blooming (DAB) and changes in peel color: $15 \mathrm{DAB}$, young fruit (YF); $30 \mathrm{DAB}$, enlarging fruit (E1); 40 DAB, E2; 55 DAB, E3; 70 DAB, E4; 85 DAB, white mature (WM); $100 \mathrm{DAB}$, beginning red (BR); 110 $\mathrm{DAB}$, half-red (HR); and $115 \mathrm{DAB}$, full-red fruit (FR). Three biological replicates of fifteen fruits were collected, and their weights were measured at each sampling period. The fruits were cut into small cubes, immediately frozen in liquid nitrogen, and stored at $80^{\circ} \mathrm{C}$ for subsequent experiments.

The fleshy fruits of 'Dongzao' in the WM stage (onset of ripening) were hand harvested from five trees per treatment. The fruits were kept at $15^{\circ} \mathrm{C}$ for $6 \mathrm{~h}$ until they were transferred to our lab, after which they were cleaned in water and then air dried for $30 \mathrm{~min}$ at room temperature $\left(20^{\circ} \mathrm{C}\right)$. Then, the fruits were randomly divided into three groups and immersed in a solution of $50 \mathrm{mg} \mathrm{L}^{-1}( \pm)$-ABA (Sigma-Aldrich), $50 \mathrm{mg} \mathrm{L}^{-1}$ NDGA (Sigma-Aldrich) or 
distilled water (control) for $10 \mathrm{~min}$. After treatment, the fruits of each group were placed in a $10 \mathrm{~L}$ uncovered plastic container and stored in darkness at $20^{\circ} \mathrm{C}$. Fruits on the 1st, 3rd, 5th, 7th and 9th days after treatment (DAT) were cut into pieces, frozen in liquid nitrogen, and stored at $-80^{\circ} \mathrm{C}$.

\section{Extraction and determination of endogenous $A B A$}

Endogenous $\mathrm{ABA}$ was extracted twice at $4{ }^{\circ} \mathrm{C}$ for $12 \mathrm{~h}$ from $1.0 \mathrm{~g}$ of crushed fruit with $4 \mathrm{~mL}$ of $80 \%$ methanol containing $200 \mathrm{mg} \mathrm{L}^{-1}$ butylated hydroxytoluene and $500 \mathrm{mg} \mathrm{L}^{-1}$ citric acid monohydrate [77]. After centrifugation, the supernatants were combined and dried under nitrogen at $35^{\circ} \mathrm{C}$. The residues were dissolved in $0.8 \mathrm{~mL}$ of $80 \%$ methanol and were filtered through a $0.22 \mu \mathrm{m}$ filter membrane.

Quantification of ABA level was modified from a previously published method [78] and performed using high-performance liquid chromatography (LC-20AT, Shimadzu, Kyoto, Japan) coupled with electrospray tandem mass spectrometry (QTRAP 5500, AB SCIEX, USA). In detail, separation of ABA was achieved using a C18 column $(2.1 \times 150 \mathrm{~mm}, 5 \mu \mathrm{m}$, Eclipse XDB, Agilent, USA) at $40^{\circ} \mathrm{C}$ with the following solvent gradient (solvent $A$, methanol; solvent $B, 0.05 \%$ formic acid): $0-8 \mathrm{~min}$, $30 \% \mathrm{~B} ; 8-15 \mathrm{~min}$, linear increase to $100 \% \mathrm{~B} ; 15-17 \mathrm{~min}$, $100 \% \mathrm{~B}$; and $17-22 \mathrm{~min}$, linear decrease to $30 \% \mathrm{~B}$, with a flow rate of $0.50 \mathrm{~mL} \mathrm{~s}^{-1}$ and an injection volume of $10.0 \mu \mathrm{L}$. For mass spectrometric analyses, the following conditions were used: IS, Turbo Spray (-), -4000 ; FP, 400; EP, - 10; CXP, - 4; CUR, 10; CAD, 5; TEM, 450; GS1, 50; GS2, 55; and ihe, ON. The retention time and mass spectrometry information concerning $\mathrm{ABA}$ were determined with a standard substance $(( \pm)$ ABA, Sigma-Aldrich), and the following conditions were ultimately selected: Q1, 263.3; Q3, 153.2; DP, -24; and $\mathrm{CE},-16$. External calibration curves were prepared using different concentrations of standard ABA dilution. Three independent biological replicates were prepared for each sample.

\section{Measurement of respiration rate and ethylene production during fruit storage}

The respiration rate was determined every day during storage at 14:00 using an LI-6400XT Photosynthesis System (Lincoln, NE, USA), which was attached to a $0.45 \mathrm{~L}$ cylindrical chamber with an air flow rate of $700 \mu \mathrm{mol} \mathrm{s}^{-1}$. The stable variation in $\Delta \mathrm{CO}_{2}$ of approximately $100 \mathrm{~g}$ of fruit was used to calculate the respiration rate [79]. Three independent biological replicates were measured for each sample.

Extraction and determination of ethylene production were performed according to our previous published method [7]. Ethylene was collected by sealing approximately $100 \mathrm{~g}$ of fruit in a $0.5 \mathrm{~L}$ glass bottle for $2 \mathrm{~h}$ at room temperature and drawing $5 \mathrm{~mL}$ of headspace gas into a $20 \mathrm{~mL}$ penicillin bottle filled with water [7]. Gas samples were collected every day during storage and determined together after collection. One milliliter of gas from the penicillin bottle was injected into a gas chromatograph (Trace GC ULTRA2010, Thermo, USA) fitted with a flame ionization detector and a Porapak 80-100 packed column $(200 \times 3 \mathrm{~mm})$. The oven, injector, and detector temperatures were 70,70 , and $150^{\circ} \mathrm{C}$, respectively. The carrier gas $\left(\mathrm{N}_{2}, \mathrm{H}_{2}\right.$, and air) flow rates were 35,35 , and $350 \mathrm{ml} \mathrm{min}^{-1}$, respectively. Three independent biological replicates were measured for each sample.

\section{Identification of genes involved in ABA biosynthesis, metabolism, and the signaling pathway}

Based on the jujube genome dataset (https://doi.org/10. 5061/dryad.83fr7) [80], the ABA biosynthesis, metabolism, and signaling pathway genes were identified and confirmed by BLASTN and SMART analyses [81]. The sequence information of these genes is listed in Additional file 10. The predicted proteins were aligned with the known protein sequences of grape, tomato, and Arabidopsis, and their sequences were subjected to KEGG analysis (https://www. genome.jp/kegg/pathway.html). Phylogenetic trees for NCED, CYP707A, BG, PYR/PYL/RCAR, PP2C, and SnRK2 genes were generated using the neighbor-joining method and 1000 bootstrap replications by MEGA 7.0 [82].

\section{RNA isolation, cDNA library construction and Illumina sequencing}

The control and ABA- and NDGA-treated fruits at 1 DAT (two biological replicates) were subjected to transcriptome sequencing. Total RNA was isolated using a plant RNA extraction kit (TaKaRa, Dalian, China) and digested with RNase-free DNase I. Thereafter, the amount of RNA was measured by spectrophotometric analysis, and its quality was further verified by agarose gel electrophoresis. A total of $3 \mu \mathrm{g}$ of RNA per sample was used for cDNA library preparation with a NEBNext Ultra RNA Library Prep Kit (NEB, USA), and the resulting PCR products were purified and quantified on an Agilent Bioanalyzer 2100 system. Sample labeling was performed on a cBot Cluster Generation System using a TruSeq PE Cluster Kit v3-cBot-HS. Afterwards, the libraries were sequenced on an Illumina HiSeq PE150 platform. The raw sequencing data were subsequently submitted to the Sequence Read Archive (https://www. ncbi.nlm.nih.gov//sra/) (Additional file 11).

Bioinformatic processing of transcriptome sequencing data Raw sequencing data generated from the Illumina HiSeq $\mathrm{X}$ platform were processed to remove low-quality reads containing adapters and a high rate of poly- $\mathrm{N}(>10 \%)$ 
using an in-house Perl script. Simultaneously, the values of the Q20, Q30, and GC content were calculated for the clean data. Afterwards, the clean data were aligned with the reference jujube genome sequences using TopHat v2.0.9 [83] and were further processed for gene expression level quantification by HTSeq v0.6.1 [84]. The relative gene expression abundance was estimated with the value of reads per kilobase of exon model per million mapped reads (RPKM) from the mean of two biological replicates [85]. For identification of differentially expressed genes (DEGs), an edgeR program [86] in OmicShare tools, a free online platform for data analysis (http://www.omic share.com/tools), was used with a fold change (FC) threshold $\geq 2$ and an false discovery rate $(F D R) \leq 0.05$. The use of edgeR allowed comparative analysis within two replicates, and it had been used in several previous papers $[12,87,88]$. The functional enrichment of DEGs was determined using Gene Ontology (GO) and pathway analysis tools within the OmicShare platform [89]. We also used MapMan 3.6.0RC1 software to enrich the putative functional annotation of the DEGs $[90,91]$.

\section{Quantitative real-time PCR validation for transcriptome expression levels}

Total RNA was extracted using a plant RNA extraction kit (TaKaRa), and $200 \mathrm{ng}$ of high-quality RNA was subsequently prepared for first-strand cDNA synthesis using a PrimeScript RT reagent kit with gDNA Eraser $(\mathrm{TaKaRa}), \mathrm{qPCR}$ was then performed using a SYBR Premix Ex Taq II kit (TaKaRa) with a total volume of $10 \mu \mathrm{L}$, which comprised $1.0 \mu \mathrm{L}$ of cDNA, $5.0 \mu \mathrm{L}$ of SYBR premix solution, $0.4 \mu \mathrm{L}$ of forward/reverse primers and $3.2 \mu \mathrm{L}$ of $\mathrm{dH}_{2} \mathrm{O}$. The thermal program for qPCR in a Roche LightCycler 96 system was set using the following conditions: $95^{\circ} \mathrm{C}$ for $30 \mathrm{~s}$; $40 \mathrm{cy}$ cles of amplification of $5 \mathrm{~s}$ at $95^{\circ} \mathrm{C}, 30 \mathrm{~s}$ at $58{ }^{\circ} \mathrm{C}$, and $30 \mathrm{~s}$ at $72^{\circ} \mathrm{C}$; and a default dissociation stage. The relative expression of each gene was normalized to that of a reference gene, $Z j U B Q$ (Zhang et al. 2015), and was ultimately calculated using the $2^{-\Delta \mathrm{Ct}}$ method (Livak and Schmittgen 2001). Sequences of the primers used for qPCR are listed in Additional file 10.

\section{Statistical analysis}

Statistical analysis was performed using the Duncan multiple range test (MRT) at the $p<0.05$ level in SPSS 19.0. The error bars in the figures represent the standard deviations of three biological replicates.

\section{Additional files}

Additional file 1: Statistic information of RNA-seq data. (XLSX $11 \mathrm{~kb})$ Additional file 2: Statistic information of mapping data. (XLSX $11 \mathrm{~kb}$ )
Additional file 3: $\mathrm{GO}$ enrichment for DEGs at DAT1. (XLSX $17 \mathrm{~kb}$ )

Additional file 4: KEGG pathway enrichment for DEGs (Level3) at DAT1. (XLSX $27 \mathrm{~kb}$ )

Additional file 5: DEGs related to metabolism pathways by MapMan. (XLSX $68 \mathrm{~kb}$ )

Additional file 6: DEGs related to hormone metabolism and signaling. (XLSX $21 \mathrm{~kb}$ )

Additional file 7: DEGs related to transcription factors involved in ripening regulation. (XLSX $27 \mathrm{~kb})$

Additional file 8: RT-qPCR validation of digital expression patterns revealed by RNA sequencing. A number of 17 genes were selected to validate the transcriptomic expressions by qPCR. The correlation coefficient between the RNA-seq data and relative expression ranged from 0.838-1.0, thereby confirming the reliability of the RNA data. (DOCX $241 \mathrm{~kb}$ )

Additional file 9: Phylogenetic analyses for NCED, CYP707A, BG, PYR/ PYL/RCAR, PP2C, and SnRK2 genes. The trees were generated by the multiple alignments with putative proteins from Arabidopsis, grape, and tomato which were uploaded in the KEGG database using MEGA 7.0. The Bootstrap value was set into 1000 (Kumar et al. 2016). (DOCX 983 kb)

Additional file 10: Sequences for ABA pathway genes. (XLSX $124 \mathrm{~kb}$ ) Additional file 11: Accession of clean reads submitted to sequence read archives (SRA) of NCBI. (XLSX $10 \mathrm{~kb}$ )

\section{Abbreviations}

AAO: Abscisic aldehyde oxidase; ABA: Abscisic acid; ABA2: xanthoxin dehydrogenase; ABA3: molybdenum cofactor sulfurtransferase; ACO: 1 aminocyclopropane-1-carboxylate oxidase; ACS: 1-aminocyclopropane-1carboxylate synthase; AHP: histidine-containing phosphotransferase; AOG: ABA-glucosyltransferase; AP2/ERF: APETALA2/ethylene-responsive element; BG: Beta-glucosidase; BR: Beginning red; CYP707A: ABA-8'hydraxylase; DAB: Day after blooming; DAT: Day after treatment; DEG: Differentially expressed gene; E: Enlarging fruit; EBF: Ethylene insensitive 3-binding F-box; EIN3/ ElL: Ethylene insensitive 3-like; ERS: Ethylene response; ETR: Ethylene receptor; FC: Fold change; FDR: False discovery rate; FR: Full-red; GA: Gibberenllin; GA20ox: GA-20-oxidase; GA20x: GA-2-oxidase; GH3: Gretchen hagen 3; GO: Gene ontology; HB: Homeobox transcription factor; HR: Half-red; HSF: Heat-shock transcription factor; KAO: ent-kaurenoic acid hydroxylase; LOG: cytokinin riboside 5'-monophosphate phosphoribohydrolase; MRT: Multiple range test; NCED: Ninecis-epoxycarotenoid dioxygenase; NDGA: Nordihydroguaiaretic acid; PP2C: Protein phosphatase 2C; PYR/PYL/RCAR: Pyrabatin resistance/pyrabatin resistance 1-like/ regulatory component; SnRK2: Sucrose nonfermenting 1-related protein kinase 2; TAA: Tryptophan aminotransferase; TF: Transcription factor; WM: White mature; YF: Young fruit; ZEP: Zeaxanthin epoxidase

\section{Acknowledgements}

Not applicable.

\section{Funding}

This work was financially supported by the National Key R\&D Program of China (2018YFD1000607), Ministry of Science and Technology of the People's Republic of China. The supporters did not play any role in the design, collection, analysis, interpretation of the relevant data, or in writing the manuscript

\section{Availability of data and materials}

All data generated or analysed during this study are included in this published article and its additional files.

\section{Authors' contributions}

$\mathrm{ZZ}$ and XL conceived and designed the experiments. ZZ performed samples preparation and conducted all the experiments, data analyses and wrote the manuscript. CK and SZ participated in determination of fruit respiration and ethylene production during postharvest storage. $\mathrm{ZZ}$ and $\mathrm{XL}$ contributed to the discussion. All authors approved the final manuscript.

Ethics approval and consent to participate Not applicable. 


\section{Consent for publication}

Not applicable.

\section{Competing interests}

The authors declare that they have no competing interests.

\section{Publisher's Note}

Springer Nature remains neutral with regard to jurisdictional claims in published maps and institutional affiliations.

\section{Author details}

${ }^{1}$ College of Forestry, Northwest A\&F University, Yangling 712100, Shaanxi, China. ${ }^{2}$ Key Comprehensive Laboratory of Forestry of Shaanxi Province, Northwest A\&F University, Yangling 712100, Shaanxi, China. ${ }^{3}$ Research Centre for Jujube Engineering and Technology of State Forestry and Grassland Administration, Northwest A\&F University, Yangling 712100, Shaanxi, China. ${ }^{4}$ Forestry Administration of Linwei District, Weinan 714000, Shaanxi, China.

\section{Received: 10 January 2019 Accepted: 26 April 2019}

\section{Published online: 08 May 2019}

\section{References}

1. Gao QH, Wu CS, Wang M. The jujube (Ziziphus jujuba mill.) fruit. A review of current knowledge of fruit composition and health benefits. J Agric Food Chem. 2013:61(14):3351-63.

2. Yao S. Past, present, and futrue of jujubes- Chinese dates in the United States. Hortscience. 2013:48(6):672-80.

3. Wu H, Wang S, Zhu J, Meng X, Wang D. Postharvest treatments affecting storage quality of Chinese jujubes. In: Liu D, editor. Chinese dates: a traditional functional food. 1st ed. Boca Raton: CRC Press; 2016. p. 272-315.

4. Cherian S, Figueroa CR, Nair H. 'Movers and shakers' in the regulation of fruit ripening. A cross-dissection of climacteric versus non-climacteric fruit. J Exp Bot. 2014;65(17):4705-22.

5. Leng P, Yuan B, Guo Y. The role of abscisic acid in fruit ripening and responses to abiotic stress. J Exp Bot. 2014;65(16):4577-88.

6. Karlova R, Chapman N, David K, Angenent GC, Seymour GB, de Maagd RA Transcriptional control of fleshy fruit development and ripening. J Exp Bot. 2014;65(16):4527-41.

7. Zhang Z, Huang J, Li X. Transcript analyses of ethylene pathway genes during ripening of Chinese jujube fruit. J Plant Physiol. 2018;224-225:1-10.

8. Sun LA, Zhang M, Ren J, Qi JX, Zhang GJ, Leng P. Reciprocity between abscisic acid and ethylene at the onset of berry ripening and after harvest. BMC Plant Biol. 2010;10(1):257.

9. Luo H, ShengJie RD, Zhang C, Ding Y, Li Z, Sun Y, Ji K, Wang Y, Li Q, et al. The role of $A B A$ in the maturation and postharvest life of a nonclimacteric sweet cherry fruit. Plant Growth Regul. 2014;33(2):373-83.

10. Wang $Y$, Wu $Y$, Duan $C$, Chen $P$, Li $Q$, Dai S, Sun L, Ji K, Sun $Y, X u$ W, et al. The expression profiling of the CSPYL, CSPP2C and CSSnRK2 gene families during fruit development and drought stress in cucumber. J Plant Physiol. 2012;169:1874-1882.

11. Wang $Y$, Guo S, Tian S, Zhang J, Ren $Y$, Sun $H$, Gong G, Zhang H, Xu Y Abscisic acid pathway involved in the regulation of watermelon fruit ripening and quality trait evolution. PLoS One. 2017;12(6):e0179944.

12. Chen J, Mao L, Lu W, Ying T, Luo Z. Transcriptome profiling of postharvest strawberry fruit in response to exogenous auxin and abscisic acid. Planta. 2016;243(1):183-97.

13. Mou W, Li D, Luo Z, Mao L, Ying T. Transcriptomic analysis reveals possible influences of ABA on secondary metabolism of pigments, flavonoids and antioxidants in tomato fruit during ripening. PLoS One. 2015;10(6):e0129598.

14. Pilati S, Bagagli G, Sonego P, Moretto M, Brazzale D, Castorina G, Simoni L, Tonelli C, Guella G, Engelen K, et al. Abscisic acid is a major regulator of grape berry ripening onset: new insights into $A B A$ signaling network. Front Plant Sci. 2017:8(1):1093.

15. Hu B, Li J, Wang D, Wang H, Qin Y, Hu G, Zhao J. Transcriptome profiling of Litchi chinensis pericarp in response to exogenous cytokinins and abscisic acid. Plant Growth Regul. 2017;84(3):437-50

16. Li D, Li L, Luo Z, Mou W, Mao L, Ying T. Comparative transcriptome analysis reveals the influence of abscisic acid on the metabolism of pigments, ascorbic acid and folic acid during strawberry fruit ripening. PLoS One. 2015;10(6):e0130037.
17. Sun L, Sun YF, Zhang M, Wang L, Ren J, Cui MM, Wang YP, Ji K, Li P, Li Q, et al. Suppression of 9-cis-epoxycarotenoid dioxygenase, which encodes a key enzyme in abscisic acid biosynthesis, alters fruit texture in transgenic tomato. Plant Physiol. 2012;158(1):283-98.

18. Wang Y, Tao X, Tang XM, Xiao L, Sun JL, Yan XF, Li D, Deng HY, Ma XR. Comparative transcriptome analysis of tomato (Solanum lycopersicum) in response to exogenous abscisic acid. BMC Genomics. 2013;14(1):841.

19. Medina-Puche L, Blanco-Portales R, Molina-Hidalgo FJ, Cumplido-Laso G, Garcia-Caparros N, Moyano-Canete E, Caballero-Repullo JL, Munoz-Blanco J, Rodriguez-Franco A. Extensive transcriptomic studies on the roles played by abscisic acid and auxins in the development and ripening of strawberry fruits. Funct Integr Genomics. 2016;16(6):671-92.

20. Mou W, Li D, Bu J, Jiang Y, Khan ZU, Luo Z, Mao L, Ying T. Comprehensive analysis of $A B A$ effects on ethylene biosynthesis and signaling during tomato fruit ripening. PLoS One. 2016:11(4):e0154072.

21. Murcia G, Pontin M, Piccoli P. Role of ABA and Gibberellin A3 on gene expression pattern of sugar transporters and invertases in Vitis vinifera CV. Malbec during berry ripening. Plant Growth Regul. 2017;84(2):275-83.

22. Estrada-Johnson E, Csukasi F, Pizarro CM, Vallarino JG, Kiryakova Y, Vioque A, Brumos J, Medina-Escobar N, Botella MA, Alonso JM, et al. Transcriptomic analysis in strawberry fruits reveals active auxin biosynthesis and signaling in the ripe receptacle. Front Plant Sci. 2017;8(1):889.

23. Wang Y, Wang Y, Ji K, Dai S, Hu Y, Sun L, Li Q, Chen P, Sun Y, Duan C, et al. The role of abscisic acid in regulating cucumber fruit development and ripening and its transcriptional regulation. Plant Physiol Biochem. 2013;64(5):70-9.

24. Kuhn N, Guan L, Dai ZW, Wu BH, Lauvergeat V, Gomes E, Li SH, Godoy F, Arce-Johnson P, Delrot S. Berry ripening: recently heard through the grapevine. J Exp Bot. 2014;65(16):4543-59.

25. Zhang M, Leng P, Zhang G, Li X. Cloning and functional analysis of 9-cisepoxycarotenoid dioxygenase (NCED) genes encoding a key enzyme during abscisic acid biosynthesis from peach and grape fruits. J Plant Physiol. 2009; 166(12):1241-52.

26. Zhao SL, Qi JX, Duan CR, Sun L, Sun YF, Wang YP, Ji K, Chen P, Dai SJ, Leng $P$. Expression analysis of the DKNCED1, DKNCED2 and DKCYP707A1 genes that regulate homeostasis of abscisic acid during the maturation of persimmon fruit. J Hortic Sci Biotechnol. 2012:87(2):165-71.

27. Ji K, Chen P, Sun L, Wang YP, Dai SJ, Li Q, Li P, Sun YF, Wu Y, Duan CR, et al. Non-climacteric ripening in strawberry fruit is linked to ABA, FaNCED2 and FaCYP707A1. Funct Plant Biol. 2012;39(4):351

28. Ji K, Kai W, Zhao B, Sun Y, Yuan B, Dai S, Li Q, Chen P, Wang Y, Pei Y, et al. SINCED1 and SICYP707A2: key genes involved in ABA metabolism during tomato fruit ripening. J Exp Bot. 2014;65(18):5243-55.

29. Jia HF, Chai YM, Li CL, Lu D, Luo JJ, Qin L, Shen YY. Abscisic acid plays an important role in the regulation of strawberry fruit ripening. Plant Physiol. 2011;157(1):188-99.

30. Nambara E, Marion-Poll A. Abscisic acid biosynthesis and metabolism. Annu Rev Plant Biol. 2005;56(1):156-85.

31. Rodrigo MJ, Alquezar B, Zacarias L. Cloning and characterization of two 9cis-epoxycarotenoid dioxygenase genes, differentially regulated during fruit maturation and under stress conditions, from orange (Citrus sinensis $\mathrm{L}$. Osbeck). J Exp Bot. 2006;57(3):633-43.

32. Hou YL, Meng K, Han Y, Ban QY, Wang B, Suo JT, LV JY, Rao JP. The persimmon 9-lipoxygenase gene DKLOX3 plays positive roles in both promoting senescence and enhancing tolerance to abiotic stress. Front Plant Sci. 2015:6(1):360-438.

33. Saito S, Hirai N, Matsumoto C, Ohigashi H, Ohta D, Sakata K, Mizutani M. Arabidopsis CYP707As encode (+)-abscisic acid 8'-hydroxylase, a key enzyme in the oxidative catabolism of abscisic acid. Plant Physiol. 2004;134(4):1439-49.

34. Cai Y, Zhu P, Liu C, Zhao A, Yu J, Wang C, Li Z, Huang P, Yu M. Characterization and expression analysis of cDNAs encoding abscisic acid 8' hydroxylase during mulberry fruit maturation and under stress conditions. Plant Cell, Tiss Org. 2016;127(1):237-49.

35. Xu ZY, Lee KH, Dong T, Jeong JC, Jin JB, Kanno Y, Kim DH, Kim SY, Seo M, Bressan RA, et al. A vacuolar beta-glucosidase homolog that possesses glucose-conjugated abscisic acid hydrolyzing activity plays an important role in osmotic stress responses in Arabidopsis. Plant Cell. 2012;24(5): 2184-99.

36. Li $Q$, Ji $K$, Sun $Y$, Luo $H$, Wang $H$, Leng $P$. The role of FaBG3 in fruit ripening and B. cinerea fungal infection of strawberry. Plant J. 2013;76(1):24-35.

37. Karppinen K, Hirvela E, Nevala T, Sipari N, Suokas M, Jaakola L. Changes in the abscisic acid levels and related gene expression during fruit 
development and ripening in bilberry (Vaccinium myrtillus L.). Phytochemistry. 2013;95(6):127-34.

38. Lin PC, Hwang SG, Endo A, Okamoto M, Koshiba T, Cheng WH. Ectopic expression of ABSCISIC ACID 2/GLUCOSE INSENSITIVE 1 in Arabidopsis promotes seed dormancy and stress tolerance. Plant Physiol. 2007;143(2): $745-58$.

39. Gonzalez-Guzman M, Abia D, Salinas J, Serrano R, Rodriguez PL. Two new alleles of the abscisic aldehyde oxidase 3 gene reveal its role in abscisic acid biosynthesis in seeds. Plant Physiol. 2004;135(1):325-33.

40. Seo M, Aoki H, Koiwai H, Kamiya Y, Nambara E, Koshiba T. Comparative studies on the Arabidopsis aldehyde oxidase (AAO) gene family revealed a major role of $\mathrm{AAO} 3$ in $\mathrm{ABA}$ biosynthesis in seeds. Plant Cell Physiol. 2004;45(11):1694-703.

41. Xiong L, Ishitani M, Lee H, Zhu JK. The Arabidopsis LOS5/ABA3 locus encodes a molybdenum cofactor sulfurase and modulates cold stress- and osmotic stress-responsive gene expression. Plant Cell. 2001;13(9):2063-83.

42. Romero P, Lafuente MT, Rodrigo MJ. The Citrus ABA signalosome: identification and transcriptional regulation during sweet orange fruit ripening and leaf dehydration. J Exp Bot. 2012;63(13):4931-45.

43. Sun L, Wang YP, Chen P, Ren J, Ji K, Li Q, Li P, Dai SJ, Leng P. Transcriptional regulation of SIPYL, SIPP2C, and SISnRK2 gene families encoding ABA signal core components during tomato fruit development and drought stress. J Exp Bot. 2011;62(15):5659-69.

44. Chai YM, Jia HF, Li CL, Dong QH, Shen YY. FaPYR1 is involved in strawberry fruit ripening. J Exp Bot. 2011;62(14):5079-89.

45. Merlot S, Gosti F, Guerrier D, Vavasseur A, Giraudat J. The AB/1 and ABI2 protein phosphatases $2 \mathrm{C}$ act in a negative feedback regulatory loop of the abscisic acid signalling pathway. Plant J. 2001;25(3):295-303.

46. Santiago J, Rodrigues A, Saez A, Rubio S, Antoni R, Dupeux F, Park SY, Marquez JA, Cutler SR, Rodriguez PL. Modulation of drought resistance by the abscisic acid receptor PYL5 through inhibition of clade a PP2Cs. Plant J. 2009;60(4):575-88.

47. Chen P, Sun YF, Kai WB, Liang B, Zhang YS, Zhai XW, Jiang L, Du YW, Leng P. Interactions of ABA signaling core components (SIPYLS, SIPP2CS, and SISnRK2s) in tomato (Solanum lycopersicon). J Plant Physiol. 2016;205(1):67-74.

48. Gambetta GA, Matthews MA, Shaghasi TH, McElrone AJ, Castellarin SD. Sugar and abscisic acid signaling orthologs are activated at the onset of ripening in grape. Planta. 2010;232(1):219-34.

49. Vlad F, Rubio S, Rodrigues A, Sirichandra C, Belin C, Robert N, Leung J, Rodriguez PL, Lauriere C, Merlot S. Protein phosphatases $2 \mathrm{C}$ regulate the activation of the Snf1-related kinase OST1 by abscisic acid in Arabidopsis. Plant Cell. 2009;21(10):3170-84.

50. Li X. Mechanisms of the signal transduction for ethylene production in apple (Malus Xdomestica) fruit development and ripening. Beijing: China Agricultural University; 2014.

51. Han Y, Dang RH, Li JX, Jiang JZ, Zhang N, Jia MR, Wei LZ, Li ZQ, Li BB, Jia WS. SUCROSE NONFERMENTING1-RELATED PROTEIN KINASE2.6, an ortholog of OPEN STOMATA1, os a negative regulator of strawberry fruit development and ripening. Plant Physiol. 2015;167(3):915-30.

52. Kou X, He Y, Li Y, Chen X, Feng Y, Xue Z. Effect of abscisic acid (ABA) and chitosan/nano-silica/sodium alginate composite film on the color development and quality of postharvest Chinese winter jujube (Zizyphus jujuba mill. cv. Dongzao). Food Chem. 2019;270:385-94.

53. Creelman RA, Bell E, Mullet JE. Involvement of a lipoxygenase-like enzyme in abscisic acid biosynthesis. Plant Physiol. 1992;99(3):1258-60

54. Wang X, Yin W, Wu J, Chai L, Yi H. Effects of exogenous abscisic acid on the expression of citrus fruit ripening-related genes and fruit ripening. Sci Hortic-Amsterdam. 2016;201(1):175-83.

55. Zhang $M$, Yuan $B$, Leng $P$. The role of ABA in triggering ethylene biosynthesis and ripening of tomato fruit. J Exp Bot. 2009;60(6):1579-88.

56. Palumbo MC, Zenoni S, Fasoli M, Massonnet M, Farina L, Castiglione F, Pezzotti M, Paci P. Integrated network analysis identifies fight-club nodes as a class of hubs encompassing key putative switch genes that induce major transcriptome reprogramming during grapevine development. Plant Cell. 2014;26(12):4617-35

57. Ramachandra Reddy A, Chaitanya KV, Vivekanandan M. Drought-induced responses of photosynthesis and antioxidant metabolism in higher plants. J Plant Physiol. 2004;161(11):1189-202.

58. Peppi M, Fidelibus M, Dokoozlian N. Application timing and concentration of abscisic acid affect the quality of 'Redglobe' grapes. J Hort Sci B. 2007:82:304-10.
59. Koyama K, Sadamatsu K, Goto-Yamamoto N. Abscisic acid stimulated ripening and gene expression in berry skins of the cabernet sauvignon grape. Funct Integr Genomics. 2010;10(3):367-81.

60. Han X, Lu W, Wei X, Li L, Mao L, Zhao Y. Proteomics analysis to understand the ABA stimulation of wound suberization in kiwifruit. J Proteome. 2018;173(1):42-51.

61. Deytieux-Belleau C, Vallet A, Doneche B, Geny L. Pectin methylesterase and polygalacturonase in the developing grape skin. Plant Physiol Biochem. 2008;46(7):638-46.

62. Huang HH, Xie SD, Xiao QL, Wei B, Zheng $\sqcup$, Wang YB, Cao Y, Zhang XG, Long TD, Hu YF et al. Sucrose and ABA regulate starch biosynthesis in maize through a novel transcription factor, ZmEREB156. Sci Rep 2016, 6:27590 (1):1.

63. Klee HJ, Giovannoni JJ. Genetics and control of tomato fruit ripening and quality attributes. Annu Rev Genet. 2011;45(1):41-59.

64. Seymour GB, Ostergaard L, Chapman NH, Knapp S, Martin C. Fruit development and ripening. Annu Rev Plant Biol. 2013;64(1):219-41.

65. Guo HW, Ecker JR. Plant responses to ethylene gas are mediated by SCF (EBF1/EBF2)-dependent proteolysis of EIN3 transcription factor. Cell. 2003;115(6):667-77.

66. Yang $Y$, Wu Y, Pirrello J, Regad F, Bouzayen M, Deng W, Li Z. Silencing SI-EBF1 and SI-EBF2 expression causes constitutive ethylene response phenotype, accelerated plant senescence, and fruit ripening in tomato. J Exp Bot. 2010;61(3):697-708.

67. McAtee P, Karim S, Schaffer R, David K. A dynamic interplay between phytohormones is required for fruit development, maturation, and ripening. Front Plant Sci. 2013;4(79):79.

68. Srivastava A, Handa AK. Hormonal regulation of tomato fruit development. A molecular perspective. J Plant Growth Regul. 2005;24(2):67-82.

69. Symons GM, Chua YJ, Ross JJ, Quittenden LJ, Davies NW, Reid JB. Hormonal changes during non-climacteric ripening in strawberry. J Exp Bot. 2012; 63(13):4741-50.

70. Kappel F, MacDonald RA. Gibberellic acid increases fruit firmness, fruit size and delays maturity of 'sweetheart' sweet cherry. J Am Pomol Soc. 2002; 56(4):210-22.

71. Wu JX, II F, Yi HL. Genome-wide identification of the transcription factors involved in Citrus fruit ripening from the transcriptomes of a late-ripening sweet orange mutant and its wild type. PLoS One. 2016;1 1(4):e0154330.

72. Zhang Z, Li XG. Genome-wide identification of AP2/ERF superfamily genes and their expression during fruit ripening of Chinese jujube. Sci Rep-Uk. 2018(8):15162.

73. Nicolas P, Lecourieux D, Kappel C, Cluzet S, Cramer G, Delrot S, Lecourieux $F$. The basic leucine zipper transcription factor ABSCISIC ACID RESPONSE ELEMENT-BINDING FACTOR2 is an important transcriptional regulator of abscisic acid-dependent grape berry ipening processes. Plant Physiol. 2014;164(1):365-83.

74. Xie XB, Li S, Zhang RF, Zhao J, Chen YC, Zhao Q, Yao YX, You CX, Zhang XS, Hao YJ. The $b H L H$ transcription factor MdbHLH3 promotes anthocyanin accumulation and fruit colouration in response to low temperature in apples. Plant Cell Environ. 2012;35(11):1884-97.

75. Zhang H, Liu YP, Wen F, Yao DM, Wang L, Guo J, Ni L, Zhang A, Tan MP, Jiang MY. A novel rice $\mathrm{C} 2 \mathrm{H} 2$-type zinc finger protein, ZFP36, is a key player involved in abscisic acid-induced antioxidant defence and oxidative stress tolerance in rice. J Exp Bot. 2014;65(20):5795-809.

76. Ma N, Feng H, Meng X, Li D, Yang D, Wu C, Meng Q. Overexpression of tomato SINAC1transcription factor alters fruit pigmentation and softening. BMC Plant Biol. 2014;14(1):351.

77. Shi WG, Li H, Liu TX, Polle A, Peng CH, Luo ZB. Exogenous abscisic acid alleviates zinc uptake and accumulation in Populus $x$ canescens exposed to excess zinc. Plant Cell Environ. 2015;38(1):207-23.

78. Pan X, Welti R, Wang X. Quantitative analysis of major plant hormones in crude plant extracts by high-performance liquid chromatography-mass spectrometry. Nat Protoc. 2010;5(6):986-92.

79. Fugate KK, Suttle JC, Campbell LG. Ethylene production and ethylene effects on respiration rate of postharvest sugarbeet roots. Postharvest Biol Tec. 2010;56(1):71-6.

80. Huang J, Zhang CM, Zhao X, Fei ZJ, Wan KK, Zhang Z, Pang XM, Yin X, Bai $Y$, Sun $X Q$, et al. The jujube genome provides insights into genome evolution and the domestication of sweetness/acidity taste in fruit trees. PLoS Genet. 2016;12(12):e1006433.

81. Letunic I, Doerks T, Bork P. SMART. Recent updates, new developments and status in 2015. Nucleic Acids Res. 2015;43(Database issue):D257-60. 
82. Kumar S, Stecher G, Tamura K. MEGA7: molecular evolutionary genetics analysis version 7.0 for bigger datasets. Mol Biol Evol. 2016;33(7):1870-4.

83. Trapnell C, Pachter L, Salzberg SL. TopHat: discovering splice junctions with RNA-Seq. Bioinformatics. 2009;25(9):1105-11.

84. Anders S, Pyl PT, Huber W. HTSeq--a Python framework to work with highthroughput sequencing data. Bioinformatics. 2015;31(2):166-9.

85. Mortazavi A, Williams BA, McCue K, Schaeffer L, Wold B. Mapping and quantifying mammalian transcriptomes by RNA-Seq. Nat Methods. 2008;5(7):621-8

86. Robinson MD, McCarthy DJ, Smyth GK. edgeR: a Bioconductor package for differential expression analysis of digital gene expression data. Bioinformatics. 2010;26(1):139-40.

87. Li X, An M, Xia Z, Bai X, Wu Y. Transcriptome analysis of watermelon (Citrullus lanatus) fruits in response to Cucumber green mottle mosaic virus (CGMMV) infection. Sci Rep-UK. 2017:7(1):16747.

88. Zhai R, Feng Y, Wang H, Zhan X, Shen X, Wu W, Zhang Y, Chen D, Dai G, Yang $Z$, et al. Transcriptome analysis of rice root heterosis by RNA-Seq. BMC Genomics. 2013;14:19.

89. Li S, Zhu S, Jia Q, Yuan D, Ren C, Li K, Liu S, Cui Y, Zhao H, Cao Y, et al. The genomic and functional landscapes of developmental plasticity in the American cockroach. Nat Commun. 2018;9(1):1008.

90. Thimm O, Blasing O, Gibon Y, Nagel A, Meyer S, Kruger P, Selbig J, Muller $L A$, Rhee SY, Stitt M. MAPMAN: a user-driven tool to display genomics data sets onto diagrams of metabolic pathways and other biological processes. Plant J. 2004;37(6):914-39.

91. Usadel B, Poree F, Nagel A, Lohse M, Czedik-Eysenberg A, Stitt M. A guide to using MapMan to visualize and compare omics data in plants: a case study in the crop species, maize. Plant Cell Environ. 2009;32(9):1211-29.

Ready to submit your research? Choose BMC and benefit from:

- fast, convenient online submission

- thorough peer review by experienced researchers in your field

- rapid publication on acceptance

- support for research data, including large and complex data types

- gold Open Access which fosters wider collaboration and increased citations

- maximum visibility for your research: over $100 \mathrm{M}$ website views per year

At $\mathrm{BMC}$, research is always in progress.

Learn more biomedcentral.com/submissions 